\title{
Does IRAS 16293-2422 have a hot core? Chemical inventory and abundance changes in its protostellar environment
}

\author{
F. L. Schöier ${ }^{1}$, J. K. Jørgensen ${ }^{1}$, E. F. van Dishoeck ${ }^{1}$, and G. A. Blake ${ }^{2}$ \\ ${ }^{1}$ Leiden Observatory, PO Box 9513, 2300 RA Leiden, The Netherlands \\ 2 Division of Geological and Planetary Sciences, California Institute of Technology, MS 150-21, Pasadena, CA 91125, USA
}

Received 18 March 2002 / Accepted 25 May 2002

\begin{abstract}
A detailed radiative transfer analysis of the observed continuum and molecular line emission toward the deeply embedded young stellar object IRAS 16293-2422 is performed. Accurate molecular abundances and abundance changes with radius are presented. The continuum modelling is used to constrain the temperature and density distributions in the envelope, enabling quantitative estimates of various molecular abundances. The density structure is well described by a single power-law falling off as $r^{-1.7}$, i.e., in the range of values predicted by infall models. A detailed analysis of the molecular line emission strengthens the adopted physical model and lends further support that parts of the circumstellar surroundings of IRAS 16293-2422 are in a state of collapse. The molecular excitation analysis reveals that the emission from some molecular species is well reproduced assuming a constant fractional abundance throughout the envelope. The abundances and isotope ratios are generally close to typical values found in cold molecular clouds in these cases, and there is a high degree of deuterium fractionation. There are, however, a number of notable exceptions. Lines covering a wide range of excitation conditions indicate for some molecules, e.g., $\mathrm{H}_{2} \mathrm{CO}, \mathrm{CH}_{3} \mathrm{OH}, \mathrm{SO}, \mathrm{SO}_{2}$ and $\mathrm{OCS}$, a drastic increase in their abundances in the warm and dense inner region of the circumstellar envelope. The location at which this increase occurs is consistent with the radius at which ices are expected to thermally evaporate off the grains. In all, there is strong evidence for the presence of a "hot core" close to the protostar, whose physical properties are similar to those detected towards most high mass protostars except for a scaling factor. However, the small scale of the hot gas and the infalling nature of the envelope lead to very different chemical time scales between low mass and high mass hot cores, such that only very rapidly produced second-generation complex molecules can be formed in IRAS 16293-2422. Alternatively, the ices may be liberated due to grain-grain collisions in turbulent shear zones where the outflow interacts with the envelope. Higher angular resolution observations are needed to pinpoint the origin of the abundance enhancements and distinguish these two scenarios. The accurate molecular abundances presented for this low-mass protostar serve as a reference for comparison with other objects, in particular circumstellar disks and comets.
\end{abstract}

Key words. stars: formation - stars: individual: IRAS 16293-2422 - ISM: abundances - stars: circumstellar matter - radiative transfer - astrochemistry

\section{Introduction}

Low mass protostars in their earliest stages of evolution are deeply embedded in large amounts of dust and gas. The nature of the emission from such star-forming regions makes them ideal to study in the infrared to radio wavelength regime. In the last decade, the sensitivity of receivers operating at these wavelengths has increased dramatically and the resulting high quality observations, supplemented by careful modelling, have provided most of the current day knowledge about the chemistry and physics, and their rapidly changing properties, in early stellar evolution. While the general scenario of low mass stellar evolution is reasonably well understood (e.g., Shu et al. 1993; Evans 1999; André et al. 2000) many uncertainties remain. For example, the nature of the hot $(T \gtrsim 90 \mathrm{~K})$ and dense $\left(n_{\mathrm{H}_{2}} \gtrsim 10^{7} \mathrm{~cm}^{-3}\right)$ regions of gas observed towards some low mass protostars is not yet fully established and the possible link

Send offprint requests to: F. L. Schöier,

e-mail: fredrik@strw.leidenuniv.nl with the so-called hot cores observed towards most high mass protostars needs to be investigated further.

In the case of high mass star formation, it has become clear that hot cores represent one of the earliest phases (Walmsley 1992). Chemically, hot cores are characterized by high abundances of fully hydrogenated molecules such as water $\left(\mathrm{H}_{2} \mathrm{O}\right)$, ammonia $\left(\mathrm{NH}_{3}\right)$ and hydrogen sulfide $\left(\mathrm{H}_{2} \mathrm{~S}\right)$, along with a rich variety of complex organic molecules ranging from methanol $\left(\mathrm{CH}_{3} \mathrm{OH}\right)$ and ethanol $\left(\mathrm{CH}_{3} \mathrm{CH}_{2} \mathrm{OH}\right)$ to methyl cyanide $\left(\mathrm{CH}_{3} \mathrm{CN}\right)$, dimethyl ether $\left(\mathrm{CH}_{3} \mathrm{OCH}_{3}\right)$, methyl formate $\left(\mathrm{HCOOCH}_{3}\right)$ and ethyl cyanide $\left(\mathrm{C}_{2} \mathrm{H}_{5} \mathrm{CN}\right)$ (Walmsley \& Schilke 1993; Kuan \& Snyder 1996; Hatchell et al. 1998; Schilke 2000). The chemical richness is explained by evaporation of the ice mantles above $\sim 90 \mathrm{~K}$, followed by rapid gasphase ion-molecule reactions leading to more complex species for a period of $\gtrsim 10^{4} \mathrm{yrs}$ (Charnley et al. 1995; Millar et al. 1997; Rodgers \& Charnley 2001, see van Dishoeck \& Blake 1998 and Langer et al. 2000 for overviews). Low mass protostars are less luminous and less massive, but a similar physical 
structure is expected except for a scale factor (Ceccarelli et al. 1996; Ivezić \& Elitzur 1997). On the other hand, shocks due to the interaction of the outflows with the envelope can also liberate ice mantles and drive a high-temperature chemistry; such shocks may be relatively more important for low-luminosity objects than for high-mass protostars (e.g., van Dishoeck et al. 1995). It is of considerable interest to establish if low mass protostars also have hot and dense regions, and if so, whether a similarly complex organic chemistry to that found in the case of high-mass protostars has ensued and whether passive heating by the accretion luminosity or active shocks dominate the liberation of grain mantles. Since it is the material in the warm inner envelope that will be incorporated into circumstellar disks, it is important to know the level of chemical complexity as it relates to forming planetary systems.

IRAS $16293-2422$ is by far the best candidate for investigating a low mass hot core (e.g., Blake et al. 1994; van Dishoeck et al. 1995; Ceccarelli et al. 2000a,b). IRAS $16293-2422$ is a deeply embedded low mass protostellar object located within the $\rho$ Ophiuchus molecular cloud complex. Due to the relative proximity of this source $(160 \mathrm{pc}$; Whittet 1974) a wealth of molecular lines has been detected, in spite of its relatively low luminosity $\left(27 L_{\odot}\right.$; Mundy et al. 1986), and this has made IRAS 16293-2422 one of the best studied young stellar objects. Interferometer observations of radio and millimetre continuum emission reveal two compact sources in the center of its circumstellar envelope (Wootten 1989; Mundy et al. 1990; Mundy et al. 1992; Looney et al. 2000), likely to be accretion disks through which matter is fed onto the central stars. The separation of the two protostars is approximately $800 \mathrm{AU}$ (Looney et al. 2000). IRAS 16293-2422 is thought to be in one of the earliest stages of formation; the observed spectral energy distribution (SED) can be fitted by a modified blackbody of $\sim 40 \mathrm{~K}$ (e.g., Walker et al. 1986; André et al. 2000) and has a high ratio of submillimetre to bolometric luminosity, suggesting a large amount of envelope mass. This places IRAS 16293-2422 among a family of deeply embedded and recently formed hydrostatic stellar objects known as, in the traditional evolutionary sequence of low mass protostellar objects (e.g., André et al. 1993), "class 0" protostars.

The circumstellar surroundings of this protobinary star were extensively studied in a large molecular line survey presented in Blake et al. (1994) and van Dishoeck et al. (1995). It was found that molecular line emission is potentially a very powerful tool to probe both the physics and chemistry of the circumstellar environment; however, a full radiative transfer analysis was not performed and the derived abundances have significant uncertainties. Due to the complexity of molecular excitation and its sensitivity to the environment, various species - and even different lines of the same species - probe different parts of the circumstellar material. At least three physically and chemically distinct parts were identified including a circumbinary envelope, circumstellar disk(s), and outflow components. The latter component was thought to be a small and warm region of a few arcsec in size where the bipolar outflow(s) interact with the inner part of the circumbinary envelope. Recently, Ceccarelli et al. (2000a,b) used deep JCMT observations of $\mathrm{H}_{2} \mathrm{CO}$ combined with a physical-chemical collapse model to argue that IRAS 16293-2422 does in fact have a hot-core-like region in which the liberation of ices is consistent with heating by the accretion luminosity.

We present here spherically-symmetric radiative transfer modelling of the dust and gas components constituting the material in the circumstellar envelope of IRAS 16293-2422. The dust parameters are constrained by the observed continuum emission in the form of submillimetre brightness maps and the SED over a large wavelength region. The resulting temperature and density structures are a prerequisite to chemical studies of the molecular gas present in the envelope. The approach taken here is different from that adopted by Ceccarelli et al. $(2000 \mathrm{a}, \mathrm{b})$ in that the physical parameters of the envelope are derived empirically from the analysis of the dust emission. Once the physical structure of the envelope is known, a detailed excitation analysis of molecular millimetre line emission is performed aimed at obtaining accurate abundances. This provides valuable insight into the complex chemistry occurring in this proto-stellar envelope. In particular, the derived abundances allow for direct comparison with other sources and comets (e.g., Bockelée-Morvan et al. 2000). Moreover, searches for evidence of abundance changes, e.g., due to evaporation of ices ("jump models") is of considerable interest. In addition to providing constraints on the chemistry, the molecular line observations give further information on the physical structure, e.g., kinematic information. Similar strategies have been adopted by van der Tak et al. (1999, 2000b) and Hogerheijde \& Sandell (2000) and have proven to be powerful tools when determining the physics and chemistry of star-forming regions.

\section{Observations and data reduction}

In this Section the observational constraints used in the radiative transfer modelling of the circumstellar envelope around IRAS 16293-2422 are presented. The SED and submillimetre continuum brightness maps constrain the physical structure of the envelope. Millimetre molecular line observations provide further information on the physical structure, in particular the large scale velocity field, and allow for studies of the chemistry in the envelope.

\subsection{Spectral energy distribution}

The SED of IRAS 16293-2422, as presented in Table 1 is used to constrain the total amount of material present in the circumstellar envelope. Care has been taken that only total fluxes integrated over the whole emitting region for wavelengths $\leq 1.3$ millimetre $(\mathrm{mm})$ are used in the SED modelling to ensure that emission from the envelope itself is dominating the observed flux. The contribution from the $\operatorname{disk}(\mathrm{s})$ to the observed emission starts to become important at wavelengths longer than $\sim 1 \mathrm{~mm}$. However, any disk emission would be significantly diluted in the relatively large beams used here (e.g., Motte \& André 2001). That thermal emission from an extended dusty envelope is responsible for the majority of the observed millimetre flux is confirmed by the radiative transfer analysis performed in Sect. 4. The model fluxes predicted by the best fit envelope model are compared with observations in Table 1. 
Table 1. The spectral energy distribution of IRAS 16293-2422.

\begin{tabular}{rccccc}
\hline \hline $\begin{array}{c}\lambda \\
{[\mu \mathrm{m}]}\end{array}$ & $\begin{array}{c}F_{\text {obs }}{ }^{\mathrm{a}} \\
{[\mathrm{Jy}]}\end{array}$ & $\begin{array}{c}\Delta F_{\text {obs }}{ }^{\mathrm{b}} \\
{[\mathrm{Jy}]}\end{array}$ & $\begin{array}{c}\theta_{\mathrm{mb}}{ }^{\mathrm{c}} \\
{\left[{ }^{\prime \prime}\right]}\end{array}$ & Ref. & $\begin{array}{c}F_{\text {mod }}{ }^{\mathrm{d}} \\
{[\mathrm{Jy}]}\end{array}$ \\
\hline 2900 & 0.60 & 0.13 & 60.0 & 3 & 0.58 \\
1300 & 6.97 & 2.24 & 30.0 & 3 & 7.5 \\
850 & 30.8 & 6.2 & 15.0 & 1 & 31.3 \\
450 & 220.4 & 44.5 & 8.8 & 1 & 220.3 \\
100 & 1032.0 & 226.0 & 237.0 & 2 & 852.4 \\
60 & 254.9 & 59.5 & 160.0 & 2 & 162.4 \\
\hline
\end{tabular}

${ }^{\text {a }}$ Observed flux integrated over emitting region.

b A calibration uncertainty of $20 \%$ has been added.

${ }^{c}$ Size of the main beam.

${ }^{\mathrm{d}}$ Flux predicted by best fit model using a density structure described by a single power-law and $\mathrm{OH} 5$ dust opacities. Note that the $60 \mu \mathrm{m}$ and $2.9 \mathrm{~mm}$ fluxes are not included in the modelling (see text for details). Refs. - (1) This paper; (2) IRAS Point Source Catalogue; (3) Walker et al. (1990).

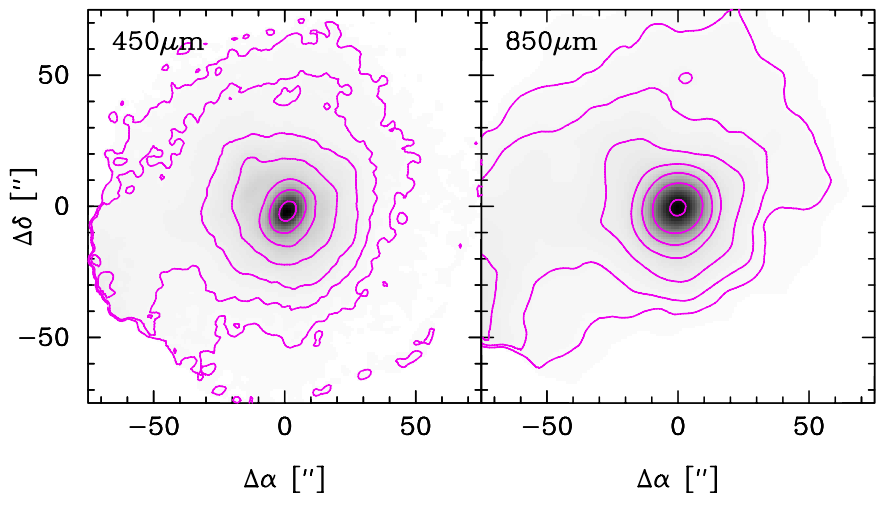

Fig. 1. SCUBA images at 450 and $850 \mu \mathrm{m}$ of IRAS 16293-2422. The contours start at the $3 \sigma$ level $\left(0.9\right.$ and $0.24 \mathrm{Jy} \mathrm{beam}^{-1}$ for 450 and $850 \mu \mathrm{m}$, respectively) and increase by multiples of 2 .

Additionally, the observed IRAS flux at $60 \mu \mathrm{m}$ is not used in the analysis since the emission at that wavelength emanates from the inner hot parts of the envelopes where the dust grain properties are probably significantly different from those in the cooler outer parts. Dust grains in the outer parts will be coated by a layer of ice which, as the temperature increases towards the star, starts to evaporate thereby changing the optical properties of the dust grains. In Sect. 4, the effect of varying the dust opacities will be investigated. A relative calibration uncertainty of $20 \%$ is added to all flux measurements, in addition to any statistical errors, which dominates the error budget and gives all points on the SED more or less equal weights.

\subsection{Submillimetre continuum observations}

Submillimetre continuum observations were retrieved from the James Clerk Maxwell Telescope (JCMT) public archive ${ }^{1}$.

\footnotetext{
${ }^{1}$ http://www.jach.hawaii.edu/JACpublic/JCMT/
}

The JCMT is operated by the Joint Astronomy Centre in Hilo, Hawaii on behalf of the present organizations: the Particle Physics and Astronomy Research Council in the United Kingdom, the National
The data were obtained during an observational run in April 1998, using the Submillimetre Common-User Bolometer Array (SCUBA), and consist of pairs of images at $450 \mu \mathrm{m}$ and $850 \mu \mathrm{m}$. The dual SCUBA array contains 91 pixels in the shortwavelength array and 37 pixels in the long-wavelength array, each covering a hexagonal 2!3 field. The SCUBA bolometer array camera is described in some detail in Holland et al. (1999).

The imaging was made using the jiggle-mapping mode to produce fully sampled maps. In this mode the SCUBA bolometers instantaneously under-sample the sky and a 64 point jiggle pattern is carried out by the telescope to fully sample both the long and short wavelength arrays. In practice, the 64 point pattern is broken down into four 16-point sub-patterns that spend $1 \mathrm{~s}$ integrating on the source at each gridpoint. After a subpattern has been completed the telescope is nodded and the pattern is repeated again so that sky subtraction can be made. In all, it takes $128 \mathrm{~s}$ to complete one full on/off source jiggle map. The jiggle mapping mode is the preferred observational mode for SCUBA when imaging sources smaller than the chop throw. Usually, a chop throw of $2^{\prime}$ is used to ensure chopping off the arrays. A larger chop throw would result in poor sky subtraction and loss in image quality.

The data were reduced in a standard way, as described in Sandell (1997), using the SCUBA reduction package SURF (Jenness \& Lightfoot 1997). The images were calibrated using simultaneous observations of Uranus retrieved from the JCMT archive. The sky opacities at $450 \mu \mathrm{m}$ and $850 \mu \mathrm{m}$ were estimated using the $1.3 \mathrm{~mm}$ opacity, monitored by the Caltech Submillimeter Observatory and listed for each individual observation, using the relations in Archibald et al. (2000). The validity of these relations have been checked and confirmed using SCUBA sky dips. The total calibration uncertainty is estimated to be approximately $\pm 20 \%$ at $450 \mu \mathrm{m}$ and about $\pm 10 \%$ at $850 \mu \mathrm{m}$. Care was taken to select data taken during good to excellent submillimetre conditions. The beam is determined from the Uranus observations and is dominated by an approximately Gaussian main beam with a deconvolved $F W H M$ of 14 '. $2 \times 16$ !' 0 and $8 . .5 \times 9$.' 1 at $850 \mu \mathrm{m}$ and $450 \mu \mathrm{m}$, respectively. A substantial error beam is, however, present at these wavelengths (see below) picking up significant amounts of flux. The error lobe pick up is estimated to be approximately $15 \%$ and $45 \%$ at $850 \mu \mathrm{m}$ and $450 \mu \mathrm{m}$, respectively, and is taken explicitly into account in the analysis.

The final $450 \mu \mathrm{m}$ and $850 \mu \mathrm{m}$ images are presented in Fig. 1. All offsets reported are relative to the adopted position of the protobinary star IRAS $16293-2422\left(\alpha_{2000}=16^{\mathrm{h}} 32^{\mathrm{m}} 22^{\mathrm{s}} .91\right.$, $\delta_{2000}=-24^{\circ} 28^{\prime} 35^{\prime \prime}$. 6 ). The locations of the two protostars IRAS 16293A (MM1) and IRAS 16293B (MM2) relative to this position are $\left(-3^{\prime \prime},-1^{\prime \prime}\right)$ and $\left(-5^{\prime \prime},+3^{\prime \prime}\right)$, respectively. The emission appears to have an overall spherical symmetry and is centered on the adopted central position within the pointing accuracy of the telescope. For comparison, the pointing accuracy of the JCMT is estimated to be about $\pm 1.5^{\prime \prime}$ in both elevation and azimuth. Also visible is a second, weak, component apparent near the eastern edge of the SCUBA maps. This

Research Council of Canada and the Netherlands Organization for Scientific Research. 
Table 2. Observational results from SCUBA images.

\begin{tabular}{ccrccc}
\hline \hline $\begin{array}{c}\lambda \\
{[\mu \mathrm{m}]}\end{array}$ & $\tau^{\mathrm{a}}$ & $\begin{array}{c}F_{\text {tot }}^{\mathrm{b}} \\
{[\mathrm{Jy}]}\end{array}$ & $\begin{array}{c}F_{\text {peak }}^{\mathrm{c}} \\
{\left[\mathrm{Jy} \mathrm{beam}^{-1}\right]}\end{array}$ & $\begin{array}{c}\mathrm{rms} \\
{\left[\mathrm{Jy} \mathrm{beam}^{-1}\right]}\end{array}$ & $\begin{array}{c}\theta_{\mathrm{mb}}^{\mathrm{d}} \\
{\left[{ }^{\prime \prime}\right]}\end{array}$ \\
\hline 450 & $0.5-0.7$ & 220.4 & 72.0 & 0.3 & 8.8 \\
850 & $0.12-0.15$ & 30.8 & 16.8 & 0.08 & 15.0 \\
\hline
\end{tabular}

${ }^{a}$ Zenith opacity of the atmosphere.

b Total flux integrated over emitting region. The calibration uncertainty is estimated to be $\sim 20 \%$.

${ }^{\mathrm{c}}$ Flux at stellar position. The calibration uncertainty is estimated to be $\sim 20 \%$.

${ }^{\mathrm{d}}$ Geometrical mean of the beam size.

second component, or IRAS $16293 \mathrm{E}\left(+77^{\prime \prime},-22^{\prime \prime}\right)$, which was first identified by its strong ammonia emission, is most probably also a class 0 protostar (Mizuno et al. 1990; Castets et al. 2001).

The $F W H M$ of the emission centered on the stellar position is $20.8 \times 19$.' 4 and $21^{\prime \prime} 9 \times 19 . ' 3$ at $850 \mu \mathrm{m}$ and $450 \mu \mathrm{m}$, respectively. The deconvolved envelope sizes assuming both the beam and brightness distribution to be described by Gaussian functions are $\sim 14^{\prime \prime}$ at $850 \mu \mathrm{m}$ and $\sim 19^{\prime \prime}$ at $450 \mu \mathrm{m}$. Thus, only the $450 \mu \mathrm{m}$ emission appears to be resolved. The observational results are summarized in Table 2. To compare the observed brightness distributions with the predictions from a spherically symmetric model, the SCUBA maps were azimuthally averaged in bins with half the corresponding beam size in width. Moreover, care was taken to block out any contribution from IRAS 16293E. The resulting radial brightness distributions are shown in Fig. 3.

\subsection{Millimetre molecular line observations}

A survey of the millimetre molecular line emission towards IRAS 16293-2422 was presented in Blake et al. (1994) and van Dishoeck et al. (1995). This large data set forms the base for the molecular excitation analysis performed in this paper. The absolute calibration uncertainty of the intensities is estimated to be $\sim 30 \%$. In addition, we have searched the JCMT public archive for complementary millimetre line observations. This additional set of data, taken at face value, is presented in Table 3. Lines for which multi-epoch observations are available in the JCMT archive typically display intensities that are consistent to $\sim 20 \%$. This was also the conclusion reached by Schöier \& Olofsson (2001) for a large survey of carbon stars. When newer data were available for a particular transition, they were usually adopted, due to a higher signal-to-noise and/or greater spectral resolution. In one case $\left[\mathrm{H}^{13} \mathrm{CO}^{+}(J=3 \rightarrow 2)\right]$ the old data set was found to have a significantly lower line intensity, possibly due to pointing problems. Additional $\mathrm{H}_{2} \mathrm{CO}$ data published recently by Loinard et al. (2000) were further used in order to increase the number of observed transitions for this molecule.

The detected molecular line emission probes the full radial range of the envelope, providing additional constraints on the physical structure of IRAS 16293-2422. Only information on the lowest transitions of the molecules, which occur at millimetre wavelengths and probe the very coldest outer parts, is lacking. The observed line shapes provide valuable information on the velocity structure in the envelope. For example, the singledish observations of abundant molecules like CO and CS typically show lines with strong self-absorption and some degree of asymmetry. The variation of the line profiles among the $\mathrm{CO}$ and CS isotopomers is potentially a sensitive probe of infall models and will be further investigated in Sect. 4.3.

\section{Radiative transfer}

In this Section the continuum and molecular line radiative transfer codes used to constrain the physical and chemical structure of the envelope around IRAS 16293-2422 are presented. The envelope is assumed to be spherically symmetric and the approach adopted here is to first determine the density and temperature structures from the dust modelling. This, in turn, allows for abundances of various molecules present in the circumstellar envelope to be determined.

\subsection{Dust radiative transfer model}

In order to model the observed continuum emission and to be able to extract some basic parameters of the dusty envelope around IRAS 16293-2422 the publically available dust radiative transfer code DUSTY ${ }^{2}$ (Ivezić et al. 1999) has been adopted. DUSTY makes use of the fact that in some very general circumstances the radiative transfer problem of the dust possesses scaling properties (Ivezić \& Elitzur 1997). The solution is presented in terms of the distance, $r / r_{i}$, scaled with respect to the inner boundary $r_{\mathrm{i}}$. In addition to the properties of the dust and the relative size of the envelope, the only parameter needed for a full description of the problem is the spectral shape of the radiation emitted by the central source. This means that the luminosity is totally decoupled from the radiative transfer problem and it is only used to scale the solution in order to obtain the absolute distance scale. This scaling property of the dust radiative transfer is very practical, in particular when modelling a large number of similar sources, and is put to use in Jørgensen et al. (2002) for a survey of low mass protostars at various evolutionary stages and in Hatchell et al. (2000) to model a selection of high mass protostars.

The most important parameter controlling the output is the dust optical depth

$\tau_{\lambda}=\kappa_{\lambda} \int_{r_{\mathrm{i}}}^{r_{\mathrm{e}}} \rho_{\mathrm{d}}(r) \mathrm{d} r$,

where $\kappa_{\lambda}$ is the dust opacity, $\rho_{\mathrm{d}}$ the density distribution of the dust in the envelope, and $r_{\mathrm{i}}$ and $r_{\mathrm{e}}$ the inner and outer radius of the dust envelope, respectively. Introducing the dust-to-gas mass ratio, $\delta$, allows Eq. (1) to be written

$\tau_{\lambda}=\kappa_{\lambda} \delta m_{\mathrm{H}_{2}} \int_{r_{\mathrm{i}}}^{r_{\mathrm{e}}} n_{\mathrm{H}_{2}}(r) \mathrm{d} r=\kappa_{\lambda} \delta \mathrm{m}_{\mathrm{H}_{2}} N_{\mathrm{H}_{2}}$

where $n_{\mathrm{H}_{2}}$ is the number density distribution of molecular hydrogen, $m_{\mathrm{H}_{2}}$ the mass of an hydrogen molecule, and $N_{\mathrm{H}_{2}}$ the

\footnotetext{
2 http://www.pa.uky.edu/ moshe/dusty/
} 
Table 3. Additional molecular line observations of IRAS 16293-2422 using the JCMT.

\begin{tabular}{|c|c|c|c|c|c|c|}
\hline Molecule & Line & $\begin{array}{c}\text { Frequency } \\
{[\mathrm{MHz}]}\end{array}$ & $\begin{array}{c}\int T_{\mathrm{mb}} \mathrm{d} V^{\mathrm{a}} \\
{\left[\mathrm{K} \mathrm{km} \mathrm{s}^{-1}\right]}\end{array}$ & $\begin{array}{c}T_{\mathrm{mb}}^{\mathrm{b}} \\
{[\mathrm{K}]}\end{array}$ & $\begin{array}{c}\Delta V^{\mathrm{b}} \\
{\left[\mathrm{km} \mathrm{s}^{-1}\right]}\end{array}$ & Ref. \\
\hline \multirow[t]{2}{*}{${ }^{13} \mathrm{CO}$} & $J=2-1$ & 220401.7 & 50.0 & 9.7: & 3.5: & 1 \\
\hline & $J=3-2$ & 330588.1 & 67.2 & 8.5: & 4.7: & 1 \\
\hline \multirow[t]{2}{*}{$\mathrm{C}^{18} \mathrm{O}$} & $J=2-1$ & 219560.4 & 21.6 & 7.8 & 2.6 & 1 \\
\hline & $J=3-2$ & 329335.0 & 33.6 & 10.1 & 3.2 & 1 \\
\hline \multirow[t]{2}{*}{$\mathrm{C}^{17} \mathrm{O}$} & $J=2-1$ & 224714.4 & 6.6 & 2.5 & 2.7 & 1 \\
\hline & $J=3-2$ & 337061.1 & 10.9 & 3.0 & 3.4 & 1 \\
\hline \multirow[t]{2}{*}{$\mathrm{CS}$} & $J=5-4$ & 244935.7 & 41.1 & 11.1 & 3.3 & 1 \\
\hline & $J=7-6$ & 342887.8 & 51.4 & 13.6 & 3.3 & 1 \\
\hline \multirow[t]{2}{*}{$\mathrm{C}^{34} \mathrm{~S}$} & $J=5-4$ & 241016.2 & 4.8 & 1.3 & 3.4 & 1 \\
\hline & $J=7-6$ & 342883.0 & 5.7 & 1.6 & 3.2 & 1 \\
\hline \multirow[t]{2}{*}{$\mathrm{HCN}$} & $J=3-2$ & 265886.4 & 51.3 & 4.0: & 7.5: & 1 \\
\hline & $J=4-3$ & 354505.5 & 63.4 & 5.0: & 7.3: & 1 \\
\hline \multirow[t]{2}{*}{ HNC } & $J=3-2$ & 271981.1 & 14.2 & 4.7: & 2.9: & 1 \\
\hline & $J=4-3$ & 362630.1 & 11.9 & 4.1: & 3.0: & 1 \\
\hline \multirow[t]{2}{*}{$\mathrm{HCO}^{+}$} & $J=3-2$ & 267557.6 & 70.3 & 18.0: & 3.5: & 1 \\
\hline & $J=4-3$ & 356734.0 & 95.9 & 21.5: & 4.0: & 1 \\
\hline \multirow[t]{2}{*}{$\mathrm{H}^{13} \mathrm{CO}^{+}$} & $J=3-2$ & 260255.5 & 10.3 & 3.8: & 2.6: & 2 \\
\hline & $J=4-3$ & 346998.5 & 8.5 & 3.1 & 2.7 & 1 \\
\hline${ }^{29} \mathrm{SiO}$ & $J=8-7$ & 342979.1 & 1.8 & 0.3 & 5.4 & 1 \\
\hline $\mathrm{HC}_{3} \mathrm{~N}$ & $J=24-23$ & 218324.8 & 0.64 & 0.15 & 3.9 & 1 \\
\hline
\end{tabular}

\footnotetext{
a Total integrated intensity calculated over full extent of line. The calibration uncertainty in the intensity scale is estimated to be $\sim 15-20 \%$.

${ }^{\mathrm{b}}$ Estimated from a Gaussian fit to the observed spectrum. A colon (:) indicates an uncertain value due to low signal-to-noise or, in the majority of cases, a significant departure from a Gaussian line profile.

Refs. - (1) JCMT public archive; (2) This paper.
}

column density of molecular hydrogen. In the derivation of Eq. (2) any possible drift velocity between the dust and the gas has been neglected. In what follows $\delta=0.01$ is assumed. The dust opacities from Ossenkopf \& Henning (1994) were used, corresponding to coagulated dust grains with thin ice mantles, at a density of $n_{\mathrm{H}_{2}} \sim 10^{6} \mathrm{~cm}^{-3}$ (Col. 5 in their Table 1, hereafter OH5). Van der Tak et al. (1999) considered various sets of dust optical properties when modelling the high mass young stellar object GL 2591, and found that models using the OH5 opacities were the only ones that gave envelope masses consistent with those derived from the modelling of the molecular line emission. In Sect. 4.2 another set of opacities will also be considered, more appropriate for regions where the ices have evaporated from the dust grains.

The dust temperature at the inner radius of the envelope is fixed to $300 \mathrm{~K}$ and this sets the inner radius. The choice of this temperature is motivated by the observations of line emission arising from highly excited molecules in the envelope. However, the inner regions are complex with breakdown of spherical symmetry and interactions between disk, envelope and outflow. In the present analysis these complications are ignored and for simplicity a smooth and spherically-symmetric envelope is assumed. Recently, Ceccarelli et al. (2000a) successfully modelled molecular line emission in the envelope around IRAS 16293-2422 down to $\sim 30 \mathrm{AU}$, assuming spherical symmetry. While the separation of the two protostars is approximately $800 \mathrm{AU}$ (Looney et al. 2000), one of the protostars IRAS 16293A (MM1) exhibits jet-like centimetre wavelength emission, water maser emission and associated millimetre molecular emission, thus appearing to be significantly more active (Wootten \& Loren 1987; Mundy et al. 1992; Schöier et al. 2002a, in prep.), which justifies the approach taken here. The central source of radiation is assumed to arise from a blackbody at $5000 \mathrm{~K}$. This is an oversimplification considering the binary nature of IRAS 16293-2422 and the uncertainty in the intrinsic SED of a protostar. The final model does not depend on the exact stellar temperature adopted, however, within a reasonable range of values, since the radiation is totally reprocessed by the circumstellar dust. The input parameters are summarized in Table 4.

The observational constraints, as presented in Sect. 2, are the SED and radial brightness distributions at $450 \mu \mathrm{m}$ and, to a lesser extent, $850 \mu \mathrm{m}$. The ability of the model to reproduce the observational constraints are quantified using the chi-squared statistic

$\chi^{2}=\sum_{i=1}^{N}\left[\frac{\left(F_{\mathrm{mod}, i}-F_{\mathrm{obs}, i}\right)}{\sigma_{i}}\right]^{2}$

where $F$ is the flux and $\sigma_{i}$ the uncertainty in observation $i$, and the summation is done over all $N$ independent observations. The radial brightness distributions from DUSTY are extended into 2D surface brightness maps and convolved with the beam as determined from planet observations. The beam convolved maps are then azimuthally averaged in $3^{\prime \prime}$ bins. In the $\chi^{2}$ fitting procedure only data points separated by one full beam are used since the $\chi^{2}$-analysis, in practice, requires uncorrelated measurements. In the analysis only the inner $50^{\prime \prime}$ of the 
Table 4. Summary of the dust radiative transfer analysis of IRAS 16293-2422 using a single power-law density distribution (see text for details).

\begin{tabular}{lr}
\hline \hline \multicolumn{2}{c}{ Fixed input parameters } \\
\hline Distance, $d$ & $160 \mathrm{pc}$ \\
Luminosity, $L$ & $27 L_{\odot}$ \\
Stellar temperature, $T_{\star}$ & $5000 \mathrm{~K}$ \\
Dust temperature at $r_{\mathrm{i}}, T_{\mathrm{d}}\left(r_{\mathrm{i}}\right)$ & $300 \mathrm{~K}$ \\
Dust opacity (OH5) at $100 \mu \mathrm{m}, \kappa_{100}$ & $86.5 \mathrm{~cm}^{2} \mathrm{~g}^{-1}$ \\
\hline \multicolumn{2}{c}{ Variable input parameters } \\
\hline Dust optical depth at $100 \mu \mathrm{m}, \tau_{100}$ \\
Density power law index, $\alpha$ \\
Envelope thickness, $r_{\mathrm{e}} / r_{\mathrm{i}}$ \\
\hline \multicolumn{2}{c}{ Best fit parameters } \\
\hline Dust optical depth at $100 \mu \mathrm{m}, \tau_{100}$ \\
Density power law index, $\alpha$ \\
Envelope thickness, $r_{\mathrm{e}} / r_{\mathrm{i}}$ & $50-6.0$ \\
\hline
\end{tabular}

Derived parameters

\begin{tabular}{lr}
\hline Inner envelope radius, $r_{\mathrm{i}}$ & $4.8 \times 10^{14} \mathrm{~cm}$ \\
Outer envelope radius, $r_{\mathrm{e}}$ & $1.2 \times 10^{17} \mathrm{~cm}$ \\
$\mathrm{H}_{2}$ column density, $N\left(\mathrm{H}_{2}\right)$ & $1.6 \times 10^{24} \mathrm{~cm}^{-2}$ \\
$\mathrm{H}_{2}$ density at $1000 \mathrm{AU}, n_{0}$ & $6.7 \times 10^{6} \mathrm{~cm}^{-3}$ \\
Envelope mass, $M_{\text {env }}$ & $5.4 M_{\odot}$ \\
Bolometric flux, $F_{\text {bol }}$ & $3.4 \times 10^{-11} \mathrm{~W} \mathrm{~m}^{-2}$ \\
\hline
\end{tabular}

brightness distributions are considered which are well above the background emission and should not be significantly affected by the $120^{\prime \prime}$ chop throw. Furthermore, the IRAS fluxes from the model were convolved with the proper filters before the $\chi^{2}$ analysis of the SED was made. The results from the dust radiative transfer are presented in Sect. 4.

\subsection{Molecular line radiative transfer model}

In order to derive accurate molecular abundances for the wealth of molecular line emission detected toward this source the detailed non-LTE radiative transfer code of Schöier (2000), based on the Monte Carlo method, was used. The code produces output that is in excellent agreement with the Monte Carlo code presented by Hogerheijde \& van der Tak (2000). It has also been tested against other molecular line radiative transfer codes, for a number of benchmark problems, to a high accuracy (van Zadelhoff et al. 2002).

Adopting the parameters of the circumstellar envelope derived from the dust radiative transfer analysis, the Monte Carlo code calculates the steady-state level populations of the molecule under study, using the statistical equilibrium equations. In the Monte Carlo method, information on the radiation field is obtained by simulating the line photons using a number of model photons, each representing a large number of real photons from all transitions considered. These model photons, emitted locally in the gas as well as injected from the boundaries of the envelope, are followed through the envelope and the number of absorptions are calculated and stored. Photons are spontaneously emitted in the gas with complete angular and frequency redistribution, i.e., the local emission is assumed to be isotropic and the scatterings are assumed to be incoherent. The weight of a model photon is continuously modified as it travels through the envelope, to take the absorptions and stimulated emissions into account. When all model photons are absorbed in, or have escaped from, the envelope the statistical equilibrium equations are solved and the whole process is then repeated until some criterion for convergence is fulfilled. Once the molecular excitation, i.e., the level populations, is obtained the radiative transfer equation can be solved exactly. The resulting brightness distribution is then convolved with the appropriate beam to allow a direct comparison with observations. In this analysis, the kinetic temperature of the gas is assumed to follow that of the dust (Ceccarelli et al. 1996; Doty \& Neufeld 1997; Ceccarelli et al. 2000a).

Typically, energy levels up to $\sim 500 \mathrm{~K}$ in the ground vibrational state were retained in the analysis. Vibrationally excited levels are not included since the radiative excitation due to the dust is generally inefficient and, for the temperature and density ranges present here, collisional excitation to these levels is negligible. Line emission from rotational transitions within vibrationally excited states has, however, been observed for some species (e.g., the CS ( $v=1, J=7 \rightarrow 6$ ) Blake et al. 1994). In Sect. 5 the nature of such emission is discussed further. Collisional rate coefficients are taken from the literature and, in the case of some linear molecules, extrapolated both in temperature and to transitions involving energy levels with higher $J$ quantum numbers when needed (see Schöier et al. 2002b, in prep. for details). For other (non-linear) species for which such extrapolations are not obvious, the excitation is assumed to be in LTE for levels for which no collisional rate coefficients are available.

In what follows, all quoted abundances, $f_{\mathrm{X}}$, for a particular molecular species $\mathrm{X}$ are relative to that of molecular hydrogen, i.e.,

$f_{\mathrm{X}}(r)=\frac{n_{\mathrm{X}}(r)}{n_{\mathrm{H}_{2}}(r)}$,

and are initially assumed to be constant throughout the envelope. Subsequently, it will be shown that in order to model the observed line emission for some molecules, e.g., $\mathrm{H}_{2} \mathrm{CO}$ and $\mathrm{SiO}$, this latter constraint has to be relaxed and a jump in $f$ needs to be introduced (see also Ceccarelli et al. 2000a,b). Van der Tak et al. (2000b) used a similar approach for the case of massive protostars.

The beam profile used in the convolution of the modelled emission is assumed to be Gaussian which is appropriate at the frequencies used here. The best fit model is estimated from the $\chi^{2}$-statistic defined in Eq. (3) using the observed integrated intensities and assuming a 30\% calibration uncertainty. Even though the new data presented in Table 3 appear to be slightly better calibrated, the old data set constitutes the vast majority of observational constraints so the larger calibration uncertainty is applied to the full data set, for simplicity. Only in the cases of $\mathrm{CO}$ and $\mathrm{CS}$ was a lower calibration uncertainty of 
$15 \%$ adopted. These molecules are regularly observed towards IRAS 16293-2422 and used as standard spectra.

\section{The physical structure of the envelope}

The envelope parameters, such as the density and temperature structures, are constrained in this Section using mainly the observed continuum emission.

\subsection{Simple analysis}

For optically thin dust emission one can derive the following expression for its intensity, in the Rayleigh-Jeans limit, as a function of the impact parameter $b$ (Shirley et al. 2000)

$\frac{I_{\nu}(b)}{I_{\nu}(0)}=\left(\frac{b}{b_{0}}\right)^{-\gamma}, \gamma=\alpha+\beta-1$,

assuming that the density

$n_{\mathrm{d}}(r) \propto r^{-\alpha}$

and temperature

$T_{\mathrm{d}}(r) \propto r^{-\beta}$

follow power-law distributions. For IRAS 16293-2422 $\gamma \sim 1.8$ is derived in the range $b \sim 15^{\prime \prime}-50^{\prime \prime}$ for the $450 \mu \mathrm{m}$ data. Assuming $\beta=0.4$ (cf., Doty \& Leung 1994; Shirley et al. 2000; see also Fig. 5), $\alpha \sim 2.4$ is obtained, consistent with the range of values determined by Shirley et al. for a sample of protostellar objects. As discussed by Shirley et al. there are a number of caveats when using this simplistic approach, e.g., the validity of applying the Rayleigh-Jeans approximation in the cool outer parts of the envelope and deviations of the dust temperature from a single power-law (see also Hogerheijde \& Sandell 2000; Doty \& Palotti 2002). Shirley et al. estimate the uncertainties in the derived $\alpha$ to be of the order \pm 0.5 using this simple approach.

\subsection{Power-law density model}

In order to derive more reliable envelope parameters the dust radiative transfer model presented in Sect. 3.1 is used. The density of the dust (and gas) is assumed to follow a simple power-law

$n_{\mathrm{H}_{2}}(r)=n_{0}\left(\frac{1000 \mathrm{AU}}{r}\right)^{\alpha}$,

where $n_{0}$ is the number density of $\mathrm{H}_{2}$ at a distance of $1000 \mathrm{AU}$ from the star. The models where the density structure is given by a single power-law will be referred to as static envelopes, since in the molecular excitation analysis no large-scale velocity field is included. The total amount of dust and its spatial distribution are simultaneously determined, i.e., $\tau_{100}$ (the optical depth at $100 \mu \mathrm{m}$ ), $\alpha$, and $r_{\mathrm{e}} / r_{\mathrm{i}}$ (the geometrical envelope thickness) are the adjustable parameters in our model. Scaling the model output to the source luminosity of $27 L_{\odot}$ and a distance of $160 \mathrm{pc}$ fixes the absolute scale and allows various physical parameters of the envelope around IRAS 16293-2422 to be determined.

To find the best fit model in the 3D parameter space the strategy adopted is to first analyze the model grid by applying the observed radial brightness distributions. As shown in Fig. 2, the $450 \mu \mathrm{m}$ and $850 \mu \mathrm{m}$ brightness distributions are sensitive to the slope of the density distribution and, to a lesser extent, the size of the dusty envelope. Changing the total amount of dust by changing $\tau_{100}$ has only a minor effect on the allowed values of $\alpha$ since normalized radial brightness distributions are used. Values of $\alpha$ in the range 1.5-1.9 are found to be acceptable, with a preferred value of 1.7. A typical accuracy of \pm 0.2 in the derived value of $\alpha$ was also found by Jørgensen et al. (2002) when analyzing SCUBA images at $450 \mu \mathrm{m}$ and $850 \mu \mathrm{m}$ for a large sample of protostars. In general, the $450 \mu \mathrm{m}$ data should provide a more reliable value of $\alpha$ because of the higher resolution, which provides a larger sensitivity to changes in the density structure.

The SED provides a good constraint on $\tau$ once the density profile is known (Fig. 2; see also Doty \& Palotti 2002). For a density slope of 1.7 the optical depth at $100 \mu \mathrm{m}$ is estimated to be approximately 4.5 . At $450 \mu \mathrm{m}$ and $850 \mu \mathrm{m}$ the optical depths are $\sim 0.4$ and $\sim 0.1$ respectively. For $\alpha \geq 1.7$ the size of the envelope is not well constrained which is not surprising given that only points in the brightness distributions out to $50^{\prime \prime}$ $\left(r_{\mathrm{e}} / r_{\mathrm{i}} \sim 250\right)$ are used. The circumstellar envelope will eventually merge with the more extended cloud material in which the object is embedded. The maximum outer radius of the envelope is fixed at the point where the dust temperature reaches $10 \mathrm{~K}$. The envelope size $r_{\mathrm{e}} / r_{\mathrm{i}}$ is estimated to be 250 for the adopted $\alpha$ of 1.7 .

The quality of the best fit model can be judged from the reduced $\chi^{2}$ obtained from

$\chi_{\mathrm{red}}^{2}=\frac{\chi_{\min }^{2}}{N-p}$,

where $p$ is the number of adjustable parameters. It is found that the best fit model presented above has $\chi_{\text {red }}^{2}=0.1$ when the SED is used as constraint and $\chi_{\text {red }}^{2}=0.4$ for the $450 \mu \mathrm{m}$ and $850 \mu \mathrm{m}$ radial brightness distributions, respectively. In all, the observations are well reproduced. The best fit solution is presented in Table 4 . The inner radius, $r_{\mathrm{i}}$, of the envelope is calculated to be $4.8 \times 10^{14} \mathrm{~cm}(32 \mathrm{AU})$ fixing the outer radius to $1.2 \times 10^{17} \mathrm{~cm}$ $\left(8000 \mathrm{AU}\right.$ or $\left.50^{\prime \prime}\right)$. Mundy et al. (1990) estimated the radial size of the envelope to be $\sim 4400 \mathrm{AU}$ from observations of ammonia emission. The best fit model is presented in Fig. 3 overplotted on the observational constraints. The total mass of molecular hydrogen contained within the outer radius of $50^{\prime \prime}$ is estimated to be $5.4 M_{\odot}$. Blake et al. (1994) derived a $\mathrm{H}_{2}$ mass content of $\sim 0.5-0.75 M_{\odot}$ within a $10^{\prime \prime}$ radius from an excitation analysis of the observed $\mathrm{C}^{17} \mathrm{O}$ molecular line emission assuming its abundance to be $3.8 \times 10^{-8}$. The present analysis gives a mass of $\sim 0.7 M_{\odot}$ within the same radius, in excellent agreement. Thus, IRAS 16293-2422 appears to have one of the most massive envelopes of the known class 0 protostars (André et al. 2000; Jørgensen et al. 2002).

Although disk emission is typically only responsible for a small fraction of the total flux at sub-millimetre wavelengths 

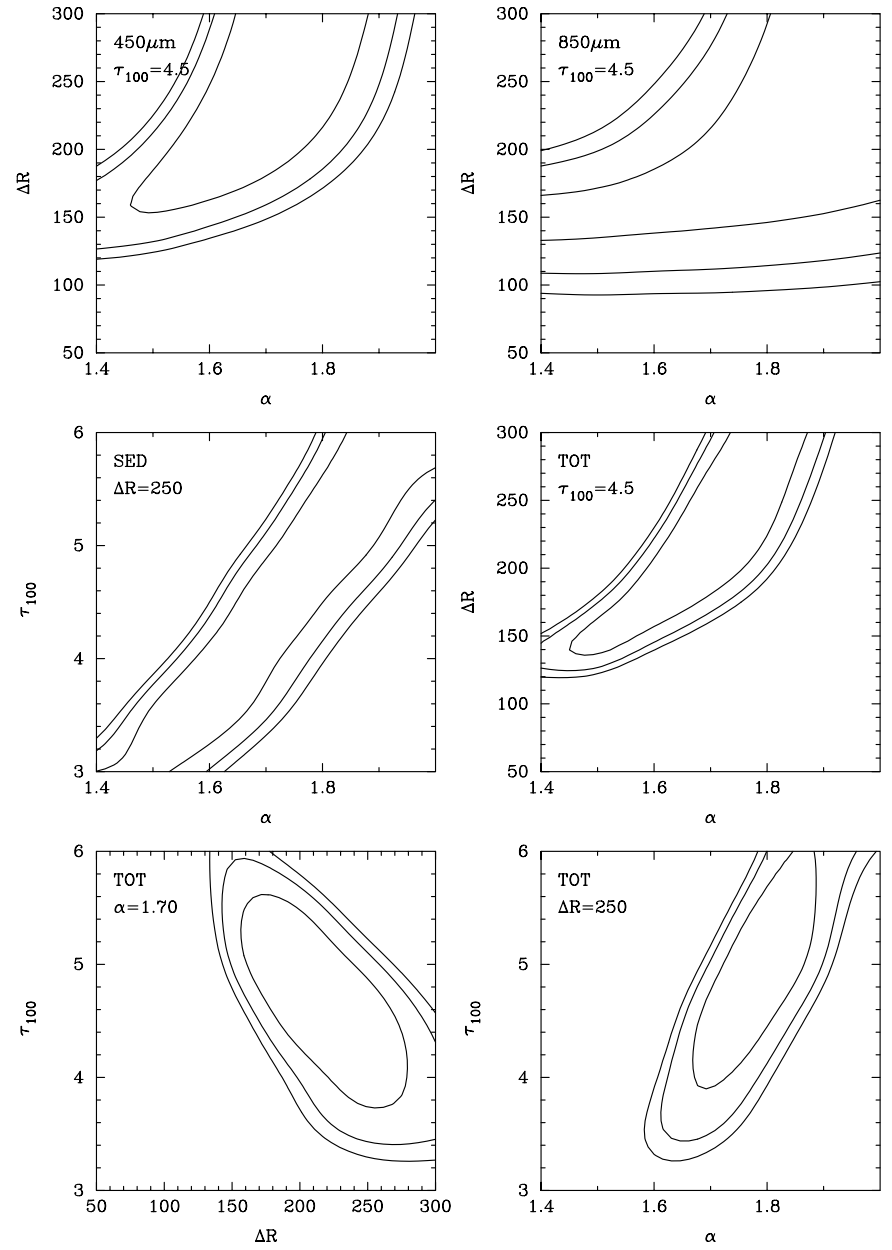

Fig. 2. $\chi^{2}$ maps showing the sensitivity of the single power-law model to the adjustable parameters $\tau_{100}, \alpha$, and $\Delta R=r_{\mathrm{e}} / r_{\mathrm{i}}$ using the SED and the SCUBA maps as observational constraints. Contours are drawn at $\chi_{\min }^{2}+(2.3,4.6,6.2)$ indicating the $68 \%$ (" $1 \sigma$ "), $90 \%$, and $95 \%$ (" $2 \sigma$ ") confidence levels, respectively.

it can contribute to the fluxes of the innermost points on the brightness profiles leading to a steeper inferred density profile. Tests in which the flux within a radius of one beam was reduced by $50 \%$ indicate that the best fit value of $\alpha$ is reduced by $0.1-0.2$.

The temperature and density structures obtained from the best fit model are presented in Fig. 5. For comparison, the predicted temperature structure based upon an optically thin approximation (Chandler \& Richer 2000) and scaled to the luminosity of IRAS 16293-2422 is shown for two different opacity laws. Both predict the dust temperature to follow a single power-law. Clearly, the temperature structure obtained from the detailed radiative transfer analysis is not well described by a power-law and has a significantly steeper gradient in the inner parts of the envelope where optical depth effects are important. The interstellar radiation field is potentially important for the temperature structure in the outer parts of the envelope. However, detailed modelling (S. Doty, priv. comm.) shows, for the envelope around IRAS 16293-2422, that this effect is small when assuming a typical interstellar radiation field. The difference in the dust temperature is $\sim 10-20 \%$ (a few $\mathrm{K}$ ) at $r \gtrsim 8 \times 10^{16} \mathrm{~cm}$.

The dust opacities adopted in the previous analysis might not be appropriate in the inner hot parts of the envelope where the ices start to evaporate off the grains. Instead opacities for bare grains, without any ice mantles, should preferably be used. DUSTY is not set up to allow dust opacities with radial dependence so only the limiting cases with or without ice mantles can be compared. To test the sensitivity of the derived envelope parameters on the adopted set of dust opacities, the analysis is repeated using the bare grain opacities presented by Ossenkopf \& Henning (1994) (Col. 2 in their Table 1; hereafter OH2). From the radial brightness distributions the same range of $\alpha$ and $\Delta R$ as when using OH5 is obtained. The optical depth, however, is significantly reduced by about a factor of two, and so is the total mass of the envelope. Adopting the model parameters as derived in Table 4, i.e., $\tau_{100}=4.5, \alpha=1.7$, and $\Delta R=250$, but using the $\mathrm{OH} 2$ opacities increases the flux at $60 \mu \mathrm{m}$ by $\sim 20 \%$ compared with the $\mathrm{OH} 5$ model. The $100 \mu \mathrm{m}$ flux is not significantly affected whereas the $1.3 \mathrm{~mm}$ flux is roughly twice that of the $\mathrm{OH} 5$ flux. A model with dust properties varying with radius (OH2 in the inner warm parts and $\mathrm{OH} 5$ in the outer cool parts) would thus serve to improve the fit to the SED. Since the bulk of the mass $(99.8 \%)$ is at low temperatures where the grains are coated with ice mantles, the model parameters obtained with the OH5 opacities were used in the chemical analysis.

In addition to the analysis of the continuum emission the observed molecular line emission is useful in constraining the physical properties of the envelope. Traditionally, $\mathrm{CO}$ and CS line emission have been extensively used for this purpose and are adopted here to test the validity of the best fit model obtained from the dust analysis. Using the radiative transfer code presented in Sect. 3.2, the total CO and CS abundances relative to $\mathrm{H}_{2}$ obtained for the best fit model presented in Table 4 are $\sim 4 \times 10^{-5}$ and $\sim 3 \times 10^{-9}$, respectively. In the $\chi^{2}$-analysis only the velocity-integrated intensities in the lines were used. These values are within a factor of about two of what is commonly derived for YSOs (van der Tak et al. 2000b). The abundances in combination with the quality of the fits (Fig. 4; see also Sect. 5), in particular the ratios among various transitions which are sensitive to the gas temperature and density, are reassuring and further strengthen the adopted physical model.

In the modelling of the molecular line emission the gas temperature is assumed to follow that of the dust. In models which self-consistently treat the energy balance the gas temperature is generally lower than that of the dust in the outer regions due to imperfect gas-grain coupling (Ceccarelli et al. 1996; Doty \& Neufeld 1997; Ceccarelli et al. 2000a). To test the effects of a departure of the gas temperature from that of the dust due to gas-grain decoupling in the outer regions, the dust temperature was scaled by a constant factor. For $T_{\text {gas }} \lessgtr 0.7 \times T_{\text {dust }}$ the envelope becomes too cool to fit the observed line intensity ratios. Thus, the gas temperature appears to follow that of the dust within $\sim 30 \%$ in the region probed by the $\mathrm{CO}$ emission.

The static envelope model fails to explain the details of the individual spectra and the potential of the molecular emission to constrain the velocity fields will be investigated further in Sect. 4.3. The derived abundances are not very sensitive to the 

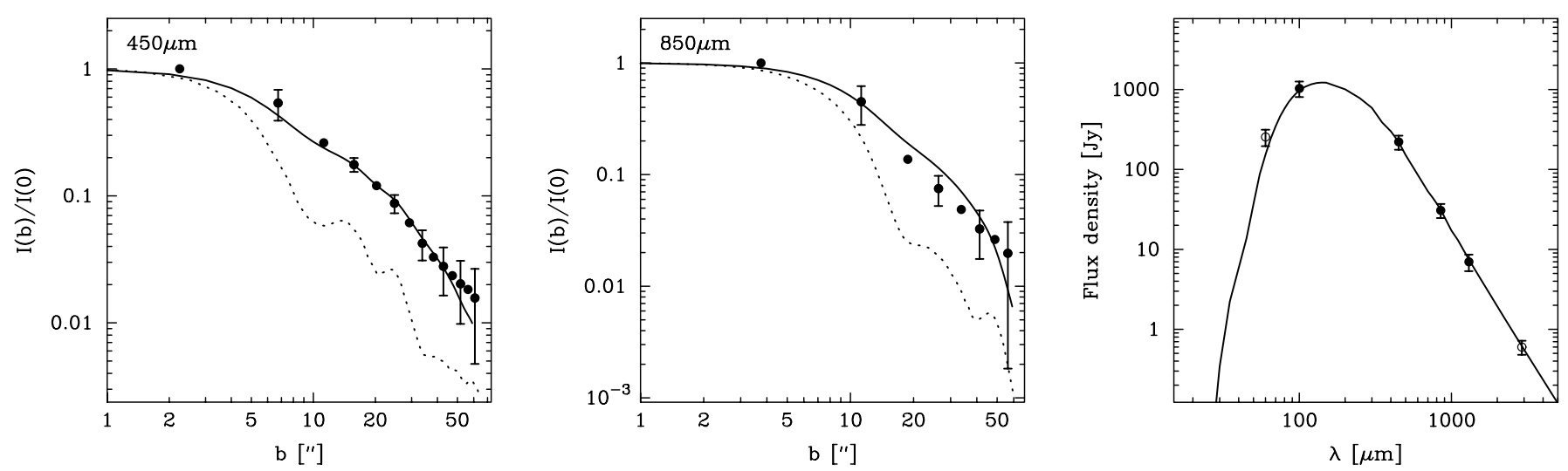

Fig. 3. Best fit model, using a density structure described by a single power-law, compared with observed radial brightness distributions and the SED. In the $\chi^{2}$-analysis of the radial brightness distributions only the data points separated by one full beam width were used, shown here with error bars. The error bars represent the rms scatter of the observations within each of the bins and are a combination of the noise as well as gradients and departure from spherical symmetry in the brightness map. The dotted line is the azimuthally averaged SCUBA beam at the time of the observation. In the SED panel the observations, represented by circles with error bars (open circles were not used in the $\chi^{2}$-analysis), are overlayed with the output from the best fit model.

adopted value of $\alpha$, within the limits derived from the dust radiative transfer model. Similarly, increasing the outer radius by a factor of two only marginally affects the line intensities. The abundances derived for a wide variety of molecular species are further presented in Sect. 5.

Thus, the emerging picture from the analysis is that the envelope around IRAS 16293-2422 indeed has a region of dense and hot gas inside a radius of $\sim 2 \times 10^{15} \mathrm{~cm}\left(150 \mathrm{AU}, 1^{\prime \prime}\right)$, with temperatures decreasing to $\sim 10 \mathrm{~K}$ at $\sim 10^{17} \mathrm{~cm}(8000 \mathrm{AU})$.

\subsection{Infall model}

Current theories of star formation state that protostars are formed from the gravitational collapse of cloud cores consisting of gas and dust (e.g., Shu et al. 1987). There is now growing evidence that low-mass protostars have parts of their circumstellar material in a state of collapse (e.g., Myers et al. 2000) and an obvious extension to the previous analysis, which uses a static envelope with a density distribution described by a single power-law, is to attempt to reproduce the results with an infall model. That the circumstellar envelope around IRAS 162932422 is in a state of collapse has been inferred previously from modelling of millimetre CS observations (Walker et al. 1986; Zhou 1995; Narayanan et al. 1998), although such conclusions based on low spatial resolution data have been questioned by Menten et al. (1987). Furthermore, Ceccarelli et al. (2000a,b) have suggested an infall model based upon a physical-chemical model. Here, the observed continuum and molecular line emission will be analyzed using the well known collapse model presented by Shu (1977).

In the Shu inside-out collapse model the self-similar solution is presented in terms of the dimensionless variable $x=$ $r / a t$, where $r$ is the radial distance scale, and characterized by the isothermal speed of sound, $a$, and the time after onset of collapse, $t$. The location of the collapsing wave front at any instant $t$ is described by $r_{\mathrm{c}}=a t$. The density $\rho$ and velocity $u$ have the form

$\rho(r, t)=\frac{\alpha(x)}{4 \pi G t^{2}}, u(r, t)=a v(x)$,

where $G$ is the gravitational constant and $\alpha(x)$ and $v(x)$ are tabulated by Shu (1977). In the static part of the envelope $(x>1)$

$\alpha(x)=\frac{2}{x^{2}}, v(x)=0$.

The asymptotic behavior as $x \rightarrow 0$ of the solution is

$$
\alpha(x)=\left(\frac{m_{0}}{2 x^{3}}\right)^{1 / 2}, v(x)=-\left(\frac{2 m_{0}}{x}\right)^{1 / 2},
$$

where $m_{0}$ is equal to 0.975 for this particular solution. Given the limited resolution provided by the JCMT at 450 and 850 microns it is not surprising that the observations are well described by a single power-law with $\alpha$ in the range $\sim 1.5-2.0$ which are the two extremes obtained from the Shu-model. The estimated value of $\alpha$ will depend on the location of the collapsing wavefront, $r_{\mathrm{c}}$. In comparison, Jørgensen et al. (2002) derived values of $\alpha$ for a large number of class 0 protostars and found values typically in the range $\sim 1.3-2.0$.

The input parameters to DUSTY are the same as for the single power-law models (see Table 4). In addition, the envelope size was fixed to a radius $5000 \mathrm{AU}$. Making the envelope larger will produce increasingly worse fits to the radial brightness distributions obtained from the SCUBA observations. The sensitivity of the two adjustable parameters $a$ and $r_{\mathrm{c}}=a t$ in the modelling is shown in Fig. 6 (left panel) where the observational constraints used are the SED and the SCUBA $450 \mu \mathrm{m}$ radial brightness distribution. The best fit model is obtained using $a \sim 0.6 \mathrm{~km} \mathrm{~s}^{-1}$ and $r_{\mathrm{c}} \sim 2 \times 10^{16} \mathrm{~cm}$, putting the age at $\sim 10^{4}$ yr.

The mass accretion rate can be estimated from

$\dot{M}=\frac{m_{0} a^{3}}{G}$, 

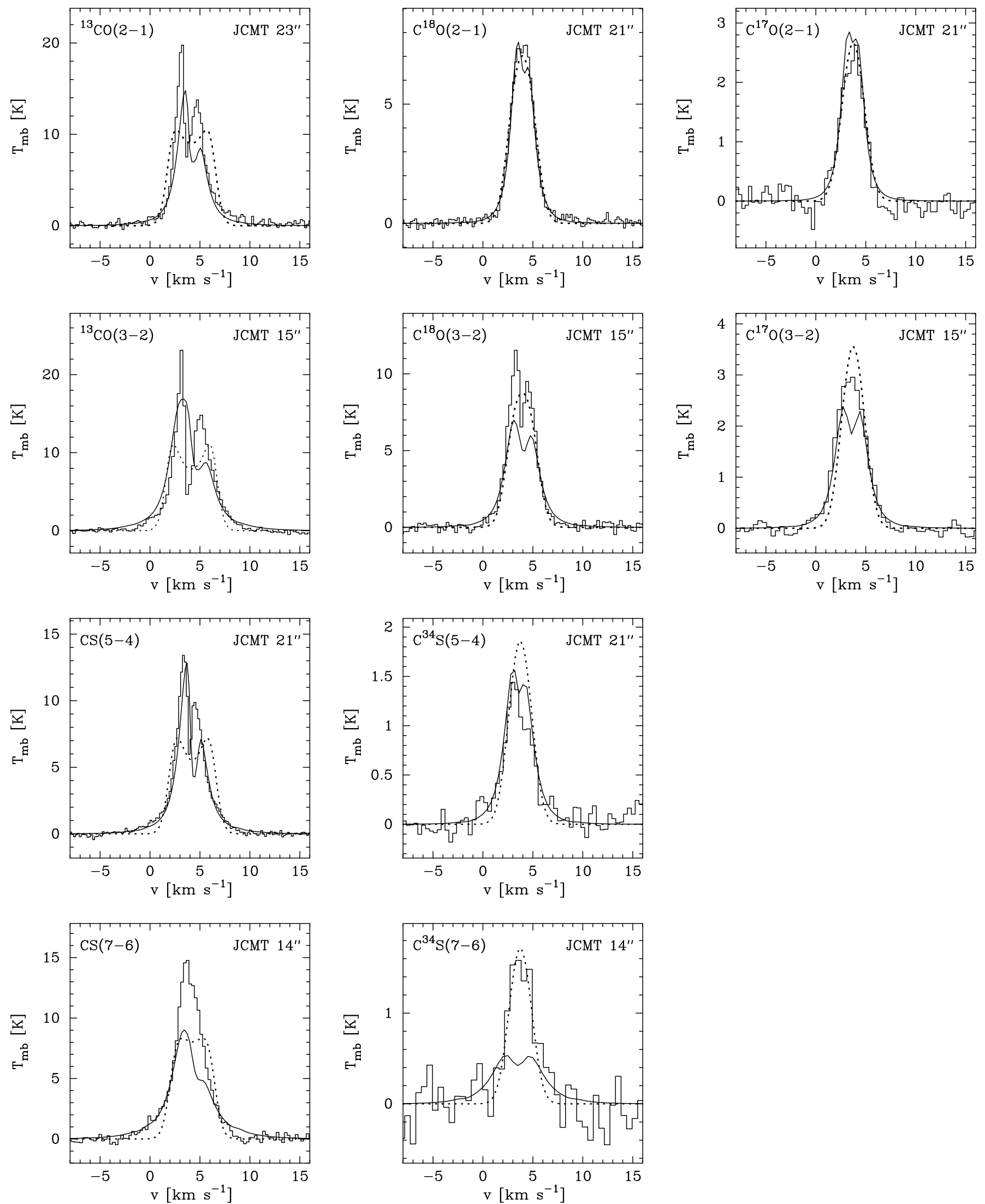

Fig. 4. Best fit CO and CS models using a static envelope (dotted line) and a Shu-type collapsing model (full line), overlayed on the observed spectra (histograms) (see text for details). The calibration uncertainty in the intensity scale for the observed spectra is $\sim 15 \%$. 

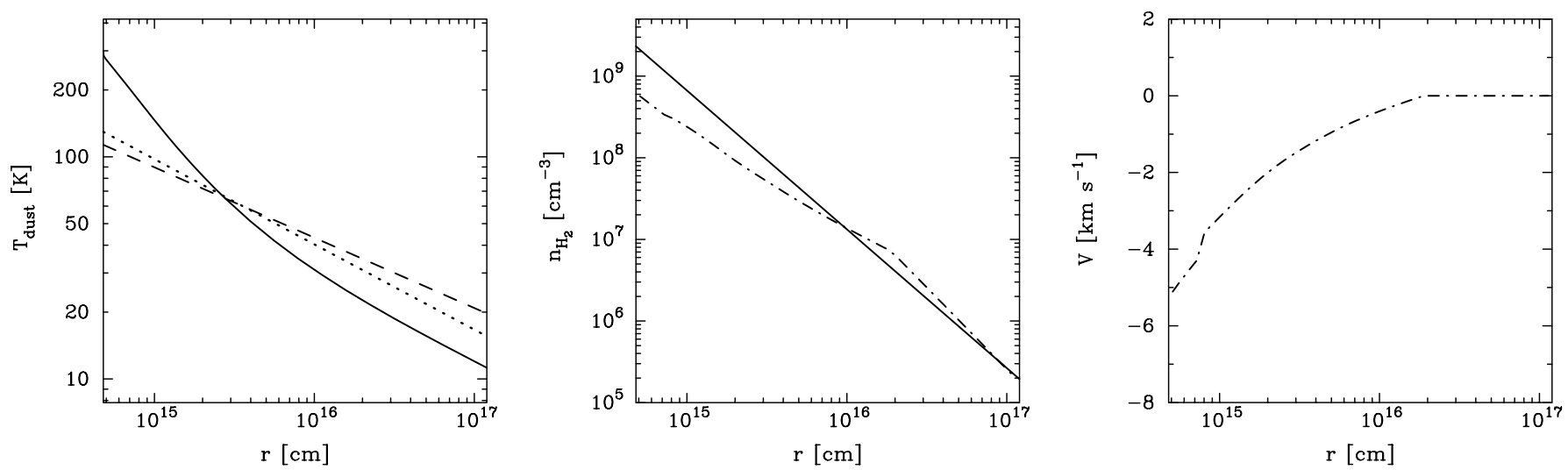

Fig. 5. Properties of the circumstellar envelope around IRAS 16293-2422 obtained from the dust modelling. Shown are the dust temperature (left) and density (middle) structures for best fit model (full line) assuming a single power-law distribution of the density. In the temperature panel, an optically thin prediction using an opacity law $\kappa_{v} \propto \nu^{\beta}$ with $\beta=1$ (dotted line; $T_{\mathrm{d}} \propto r^{-0.4}$ ) and $\beta=2$ (dashed line; $T_{\mathrm{d}} \propto r^{-0.33}$ ), is also shown. In the density panel the best fit Shu-type collapsing envelope model is also shown (dash-dotted line). The velocity structure obtained from the Shu infall model is shown in the right panel.
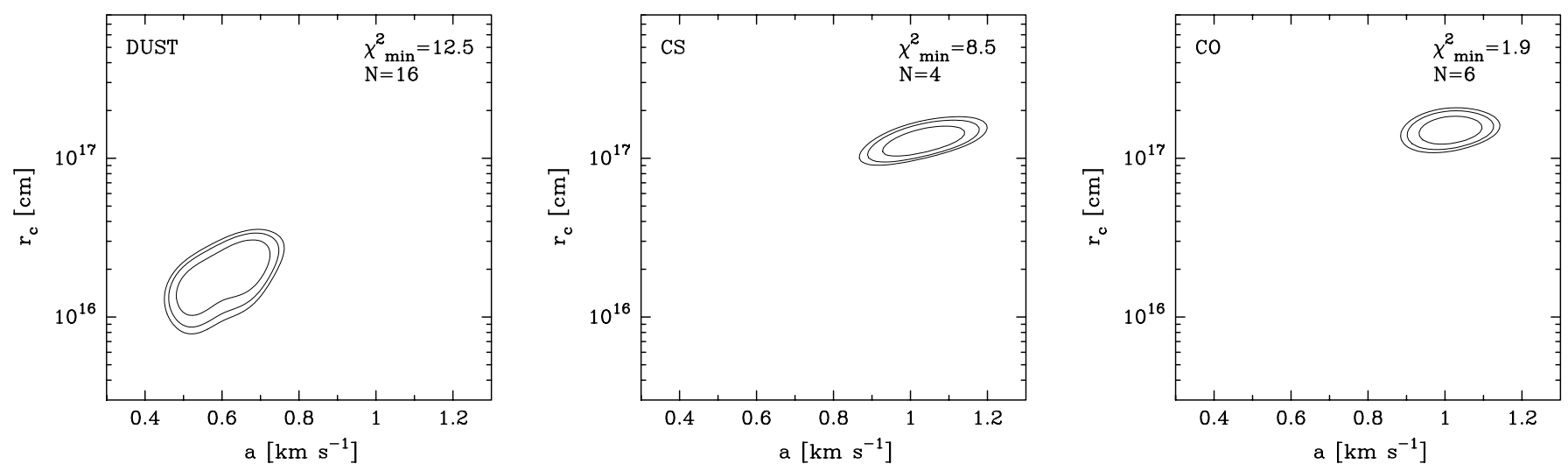

Fig. 6. $\chi^{2}$-maps showing the sensitivity of the Shu-collapse model to the adjustable parameters in the modeling of the dust continuum emission (left) and the $\mathrm{CS}\left(\mathrm{CS}\right.$ and $\mathrm{C}^{34} \mathrm{~S}$ transitions; centre) and $\mathrm{CO}\left({ }^{13} \mathrm{CO}, \mathrm{C}^{17} \mathrm{O}\right.$, and $\mathrm{C}^{18} \mathrm{O}$ transitions; right) line emission compared to observations. Contours are drawn at $\chi_{\min }^{2}+(2.3,4.6,6.2)$ indicating the $68 \%$ (" $1 \sigma$ "), $90 \%$, and $95 \%$ (" $2 \sigma$ ") confidence levels, respectively. The number of observational constraints used, $N$, are also shown. The quality of the best fit model can be estimated from the reduced chi-squared statistic $\chi_{\text {red }}^{2}=\chi_{\min }^{2} /(N-2)$.

where $G$ is the gravitational constant. For the best fit model $\dot{M} \sim 5 \times 10^{-5} M_{\odot} \mathrm{yr}^{-1}$ is obtained. Such a high accretion rate is able to account for the source luminosity. The total mass contained in the envelope within $5000 \mathrm{AU}$ is $\sim 3.3 M_{\odot}$, consistent with the estimate obtained from the static envelope model within the same radius.

The relative success of the dust modelling using a static envelope, with a single power-law to describe the density structure, makes it hard to discriminate between the two models in the present analysis. In Fig. 5 the density and velocity structures obtained from the best fit Shu model are presented and, for the density, compared with results from the static envelope model. The largest discrepancy occurs at small radial distances where the collapsing envelope model predicts about a factor of two to three lower densities. We stress the observational data set used in the dust modelling is not directly probing this region. At larger radii the model is better constrained and the two models agree well. It should be noted that the best fit single power-law model gives slightly better reduced $\chi^{2}$ values for the combined set of observations. However, the molecular data provide further constraints since they have the potential to probe the large scale velocity field.

In Fig. 4, spectra of $\mathrm{CO}$ and CS line emission as observed with the JCMT are presented. Lines which are optically thick, like $\mathrm{CS},{ }^{13} \mathrm{CO}$ and $\mathrm{C}^{18} \mathrm{O}(J=3 \rightarrow 2)$, show a distinct, narrow, absorption feature near the stellar velocity. This feature is due to effective self-absorption in the outer cool parts of the envelope. Also, the degree of the asymmetry in the line profiles increases with the optical depth in the lines. In the optically thin lines the self-absorption feature disappears and the lines are well described by a single Gaussian profile. The width of the self-absorption feature constrains the turbulent velocity to $\sim 0.3 \mathrm{~km} \mathrm{~s}^{-1}$ in the outer envelope. For simplicity this value is adopted throughout the envelope. A turbulent velocity component varying with radius is beyond the scope of this article, see however Stark et al. (2002, in prep.).

The observed CS emission (including that from $\mathrm{C}^{34} \mathrm{~S}$ ) is analyzed using the abundances derived from the static envelope model presented in Sect. 4.2 (see also Sect. 5). From only the integrated intensities it is possible to constrain both $a$ and $r_{\mathrm{c}}$ 
as is shown in Fig. 6 (middle panel). The model spectra are presented in Fig. 4 together with the observations. The fit to the integrated intensities is worse than that obtained from the single power-law model presented in Sect. 4.2, however. In particular the $C^{34} S(J=7 \rightarrow 6)$ line is poorly reproduced in the infall model. Using also the line profiles as constraints we find that a model where the collapsing wavefront is located at $\sim 1.0 \times$ $10^{17} \mathrm{~cm}$ and the value of $a$ is $\sim 0.9 \mathrm{~km} \mathrm{~s}^{-1}$ best reproduces the observations.

Analyzing the $\mathrm{CO}$ emission $\left({ }^{13} \mathrm{CO}, \mathrm{C}^{18} \mathrm{O}\right.$, and $\left.\mathrm{C}^{17} \mathrm{O}\right)$ gives yet another set of estimates. At first the abundances derived from the static envelope models presented in Sect. 4.2 (see also Sect. 5) are adopted. From the analysis of the integrated intensities shown in Fig. 6 (right panel) and the line profiles presented in Fig. 4 a Shu-model with $a \sim 1.0 \mathrm{~km} \mathrm{~s}^{-1}$ and $r_{\mathrm{c}} \sim$ $1.2 \times 10^{17} \mathrm{~cm}$ reproduces the $\mathrm{CO}$ observations well, in excellent agreement with the CS modelling. The derived dynamical age of the system is $\sim 3 \times 10^{4} \mathrm{yr}$. In contrast with the CS modelling, the fit to the integrated $\mathrm{CO}$ intensities is equally good as obtained for the single power-law model. As discussed in Sect. 5, the $\mathrm{CO}$ abundance obtained from the static envelope model is about a factor of 2-3 lower than what is typically observed for interstellar gas. If instead the $\mathrm{CO}$ abundance relative to $\mathrm{H}_{2}$ is assumed to be the "standard" interstellar value of $1 \times 10^{-4}$ and the standard isotopic ratios are assumed $\left(\left[\mathrm{CO} /{ }^{13} \mathrm{CO}\right]=60\right.$, $\left[\mathrm{CO} / \mathrm{C}^{17} \mathrm{O}\right]=2500$, and $\left.\left[\mathrm{C}^{18} \mathrm{O} / \mathrm{C}^{17} \mathrm{O}\right]=3.9\right)$ the estimate of $a$ is $\sim 0.75$ and $r_{\mathrm{c}} \sim 3 \times 10^{17} \mathrm{~cm}$. However, the quality of the fit becomes worse in this case.

The results obtained from the best fit Shu-model are presented in Fig. 4 overlayed onto the observed CO and CS spectra. The integrated intensities and, to some extent, line profiles can be modelled with the spherically symmetric infall solution. The details of the spectra will, however, intricately depend on the adopted geometry, velocity fields, and chemical gradients. The velocity field, in particular position-velocity maps of the source, form a stringent test of the dynamical models. For IRAS $16293-2422$ it will likely be necessary to include a rotational component to the velocity field (Menten et al. 1987; Zhou 1995; Narayanan et al. 1998).

Other estimates of the infall radius, based on analysis of molecular line emission, range between about 5 and $15 \times$ $10^{16} \mathrm{~cm}$ (Walker et al. 1986; Zhou 1995; Narayanan et al. 1998; Ceccarelli et al. 2000a) in excellent agreement with the values obtained here from analysis of CO and CS emission. However, there appears to be a discrepancy between the dust and molecular line analysis, possibly reflecting the fact that the simple Shu-collapsing core model is not fully adequate to describe the state of the infalling material and/or that some of the $\mathrm{CO}$ and CS emission is associated with the outflow and surrounding cloud. Moreover, gradients in the molecular abundances will affect the parameters derived.

\section{Molecular abundances}

The basic envelope parameters derived from the dust radiative transfer modelling performed in Sect. 4, in particular the density and temperature distributions, are used as input for the Monte Carlo modelling of the molecular line emission.
The static power-law model is adopted; the abundances obtained with the best fitting infall model generally differ by no more than $\sim 25 \%$ for a constant abundance model. However, models where a drastic enhancement in the abundance is introduced ("jump-models") require 2-3 times larger abundances in the inner hot part for a Shu-type collapsing model, reflecting the significantly lower density compared to the static powerlaw model in this region (Fig. 5). Changing the envelope parameters describing the static power-law model within the accepted range of values (Fig. 2) typically affects the abundances obtained for the best fit model by less than $\pm 25 \%$ for constant abundance models. In "jump-models" the effect on lines which are sensitive to the conditions in the innermost dense and hot regions can be higher, up to $\pm 50 \%$.

\subsection{Constant abundance models}

The abundances are initially assumed to be constant throughout the envelope. For simplicity the observed lines are assumed to be broadened (in addition to thermal line broadening) by microturbulent motions only. The microturbulent velocity is set equal to $2 \mathrm{~km} \mathrm{~s}^{-1}$ throughout the envelope typically producing lines $\sim 4 \mathrm{~km} \mathrm{~s}^{-1}(F W H M)$ wide (see Sect. 6). The presence of a global velocity field, e.g., infall, outflow, or rotation, would serve to reduce the optical depths of the line emission, so that the abundances presented in Table 5 are strictly lower limits. However, such effects will not significantly increase the inferred abundances since they are largely derived from optically thin lines. The observational constraints used in the modelling are the total velocity-integrated line intensities.

In many cases the observations are well reproduced assuming a constant abundance throughout the envelope, as seen from their reduced $\chi^{2} \sim 1$. In the cases where the fits are good the derived abundances are generally consistent with typical values found in quiescent molecular clouds. In addition, the isotopic ratios of ${ }^{18} \mathrm{O} /{ }^{17} \mathrm{O} \sim 3.9$ determined from $\mathrm{CO}$ observations and ${ }^{32} \mathrm{~S} /{ }^{34} \mathrm{~S} \sim 25$ from CS observations, agree well with interstellar values (Wilson \& Rood 1994). A notable exception is the relatively low abundance, $\sim 7 \times 10^{-11}$, derived for HNC. The abundances derived by Blake et al. (1994) and van Dishoeck et al. (1995) agree surprisingly well with the new, more accurate, estimates presented here (Table 5), typically within a factor of $\sim 2$. Those abundances were derived from statistical equilibrium equations assuming a constant temperature and density. The agreement indicates that the adopted values were representative of the region from which most of the submillimetre emission arises.

For the main isotopes of $\mathrm{HCN}$ and $\mathrm{HCO}^{+}$the emission is highly optically thick and the models are relatively insensitive to the molecular abundance. The abundances of these molecules were instead estimated from the rarer isotopomers assuming a standard isotope ratio, i.e., ${ }^{12} \mathrm{C} /{ }^{13} \mathrm{C}=60$. The abundances obtained in this way fail to account for all of the observed flux in the $\mathrm{HCN}$ and $\mathrm{HCO}^{+}$lines, as evidenced by their high reduced $\chi^{2}$-values in Table 5. Adopting the best fit Shuinfall model derived from $\mathrm{CO}$ and CS observations and presented in Sect. 4.3 introduces a large-scale velocity field which 
Table 5. Derived abundances using a constant molecular abundance $f_{X}$ relative to $\mathrm{H}_{2}$ throughout the envelope.

\begin{tabular}{|c|c|c|c|c|c|c|c|}
\hline & Molecule & $f_{X}$ & $\chi_{\text {red }}^{2}$ & $N^{\mathrm{b}}$ & $f_{X}^{\text {old }^{c}}$ & $\begin{array}{c}E_{\min }{ }^{\mathrm{d}} \\
{[\mathrm{K}]}\end{array}$ & $\begin{array}{c}E_{\max }{ }^{\mathrm{e}} \\
{[\mathrm{K}]}\end{array}$ \\
\hline${ }^{13} \mathrm{CO}$ & carbon monoxide & $6.5 \times 10^{-7}$ & 0.2 & 3 & $1.6 \times 10^{-6} \dagger$ & 15.9 & 31.7 \\
\hline $\mathrm{C}^{18} \mathrm{O}$ & & $6.2 \times 10^{-8}$ & 0.5 & 2 & $1.0 \times 10^{-7}$ & 15.8 & 31.6 \\
\hline $\mathrm{C}^{17} \mathrm{O}$ & & $1.6 \times 10^{-8}$ & 0.1 & 2 & $3.8 \times 10^{-8}$ & 16.2 & 32.4 \\
\hline $\mathrm{HCO}^{+}$ & formyl ion & $1.4 \times 10^{-9} \dagger$ & 10.9 & 3 & $1.8 \times 10^{-9} \dagger$ & 25.7 & 42.8 \\
\hline $\mathrm{H}^{13} \mathrm{CO}^{+}$ & & $2.4 \times 10^{-11}$ & 0.1 & 3 & $7.5 \times 10^{-12}$ & 25.0 & 41.6 \\
\hline $\mathrm{HC}^{18} \mathrm{O}^{+}$ & & $6.4 \times 10^{-12}$ & $\ldots$ & 1 & $3.5 \times 10^{-12}$ & 40.9 & 40.9 \\
\hline $\mathrm{DCO}^{+}$ & & $1.3 \times 10^{-11}$ & 0.3 & 2 & $1.5 \times 10^{-11}$ & 20.7 & 51.9 \\
\hline $\mathrm{CN}$ & cyanogen & $8.0 \times 10^{-11}$ & 1.2 & 4 & $1.0 \times 10^{-10}$ & 16.3 & 32.7 \\
\hline $\mathrm{HCN}$ & hydrogen cyanide & $1.1 \times 10^{-9} \dagger$ & 2.3 & 3 & $1.9 \times 10^{-9} \dagger$ & 25.5 & 42.5 \\
\hline $\mathrm{H}^{13} \mathrm{CN}$ & & $1.8 \times 10^{-11}$ & 6.2 & 2 & $1.4 \times 10^{-11}$ & 24.9 & 41.4 \\
\hline $\mathrm{HC}^{15} \mathrm{~N}$ & & $7.6 \times 10^{-12}$ & $\cdots$ & 1 & $7.0 \times 10^{-12}$ & 24.8 & 24.8 \\
\hline DCN & & $1.3 \times 10^{-11}$ & 3.5 & 2 & $2.5 \times 10^{-11}$ & 20.9 & 52.1 \\
\hline $\mathrm{HNC}$ & hydrogen isocyanide & $6.9 \times 10^{-11}$ & 0.2 & 3 & $1.5 \times 10^{-10}$ & 26.1 & 43.5 \\
\hline $\mathrm{HN}^{13} \mathrm{C}$ & & $5.2 \times 10^{-12}$ & $\cdots$ & 1 & $2.5 \times 10^{-12}$ & 25.1 & 25.1 \\
\hline DNC & & $4.2 \times 10^{-12}$ & 3.0 & 2 & $5.0 \times 10^{-12}$ & 22.0 & 36.6 \\
\hline $\mathrm{HC}_{3} \mathrm{~N}$ & cyanoacetylene & $1.5 \times 10^{-10}$ & 1.8 & 3 & $2.5 \times 10^{-11}$ & 131.0 & 177.3 \\
\hline $\mathrm{CH}_{3} \mathrm{CN}$ & methyl cyanide & $1.4 \times 10^{-10}$ & 5.0 & 7 & $1.5 \times 10^{-10}$ & 68.9 & 257.9 \\
\hline $\mathrm{HNCO}$ & isocyanic acid & $1.3 \times 10^{-10}$ & 1.0 & 2 & $1.7 \times 10^{-10}$ & 112.6 & 126.6 \\
\hline $\mathrm{C}_{2} \mathrm{H}$ & ethynyl & $2.1 \times 10^{-10}$ & 0.3 & 4 & $2.5 \times 10^{-10}$ & 25.1 & 62.9 \\
\hline $\mathrm{C}_{2} \mathrm{D}$ & & $3.5 \times 10^{-11}$ & 0.0 & 2 & $4.5 \times 10^{-11}$ & 20.7 & 20.7 \\
\hline $\mathrm{C}_{3} \mathrm{H}_{2}$ & cyclopropenylidene & $1.6 \times 10^{-11}$ & 4.1 & 6 & $3.5 \times 10^{-11}$ & 19.5 & 86.9 \\
\hline $\mathrm{CH}_{3} \mathrm{C}_{2} \mathrm{H}$ & methyl acetylene & $1.4 \times 10^{-9}$ & 2.7 & 6 & $6.5 \times 10^{-10}$ & 74.6 & 163.2 \\
\hline $\mathrm{o}-\mathrm{H}_{2} \mathrm{CO}$ & formaldehyde & $6.9 \times 10^{-10}$ & 4.1 & 9 & $5.2 \times 10^{-10}$ & 21.9 & 174.0 \\
\hline $\mathrm{p}-\mathrm{H}_{2} \mathrm{CO}$ & & $5.1 \times 10^{-10}$ & 2.9 & 8 & $1.8 \times 10^{-10}$ & 21.0 & 240.7 \\
\hline $\mathrm{o}-\mathrm{H}_{2}{ }^{13} \mathrm{CO}$ & & $1.2 \times 10^{-11}$ & 3.7 & 4 & $\cdots$ & 21.7 & 61.3 \\
\hline $\mathrm{p}-\mathrm{H}_{2}{ }^{13} \mathrm{CO}$ & & $9.5 \times 10^{-12}$ & 3.6 & 2 & $\cdots$ & 51.1 & 98.4 \\
\hline o-HDCO & & $1.0 \times 10^{-10}$ & 0.1 & 2 & $7.3 \times 10^{-11}$ & 52.4 & 56.3 \\
\hline p-HDCO & & $5.1 \times 10^{-11}$ & 5.0 & 2 & $2.5 \times 10^{-11}$ & 30.8 & 62.7 \\
\hline $\mathrm{CH}_{3} \mathrm{OH}$ & methanol & $1.7 \times 10^{-9}$ & 5.7 & 23 & $4.4 \times 10^{-9}$ & 15.5 & 187.6 \\
\hline $\mathrm{CH}_{2} \mathrm{CO}$ & ketene & $5.3 \times 10^{-10}$ & 9.2 & 2 & $1.8 \times 10^{-10}$ & 88.0 & 160.0 \\
\hline $\mathrm{HCOOH}$ & formic acid & $<3.0 \times 10^{-10}$ & $\cdots$ & $\cdots$ & $<3.0 \times 10^{-10}$ & $\cdots$ & $\ldots$ \\
\hline $\mathrm{CH}_{3} \mathrm{CHO}$ & acetaldehyde & $<6.0 \times 10^{-11}$ & $\cdots$ & $\cdots$ & $<1.0 \times 10^{-10}$ & $\cdots$ & $\cdots$ \\
\hline $\mathrm{CH}_{3} \mathrm{OCH}_{3}$ & dimethyl ether & $<6.0 \times 10^{-10}$ & $\cdots$ & $\ldots$ & $<2.0 \times 10^{-9}$ & $\ldots$ & $\ldots$ \\
\hline $\mathrm{HCOOCH}_{3}$ & methyl formate & $<5.0 \times 10^{-9}$ & $\cdots$ & $\cdots$ & $\ldots$ & $\cdots$ & $\cdots$ \\
\hline $\mathrm{CS}$ & carbon monosulfide & $3.0 \times 10^{-9}$ & 0.5 & 3 & $1.1 \times 10^{-9}$ & 35.3 & 65.8 \\
\hline $\mathrm{C}^{34} \mathrm{~S}$ & & $1.2 \times 10^{-10}$ & 0.9 & 3 & $5.0 \times 10^{-11}$ & 34.7 & 64.8 \\
\hline $\mathrm{SO}$ & sulfur monoxide & $4.4 \times 10^{-9}$ & 2.4 & 9 & $3.9 \times 10^{-9}$ & 35.0 & 87.5 \\
\hline $\mathrm{SO}_{2}$ & sulfur dioxide & $6.2 \times 10^{-10}$ & 6.1 & 10 & $1.5 \times 10^{-9}$ & 19.2 & 82.5 \\
\hline OCS & carbonyl sulfide & $7.0 \times 10^{-9}$ & 6.5 & 2 & $7.1 \times 10^{-9}$ & 122.6 & 237.0 \\
\hline $\mathrm{HCS}^{+}$ & thioformyl ion & $2.4 \times 10^{-11}$ & 7.0 & 2 & $2.0 \times 10^{-11}$ & 30.7 & 73.7 \\
\hline $\mathrm{O}^{13} \mathrm{CS}$ & & $6.7 \times 10^{-10}$ & 5.6 & 2 & $2.8 \times 10^{-10}$ & 99.5 & 122.2 \\
\hline $\mathrm{OC}^{34} \mathrm{~S}$ & & $1.6 \times 10^{-9}$ & 1.8 & 3 & $3.2 \times 10^{-10}$ & 99.8 & 147.7 \\
\hline $\mathrm{H}_{2} \mathrm{~S}$ & hydrogen sulfide & $1.6 \times 10^{-9}$ & $\cdots$ & 1 & $1.5 \times 10^{-9}$ & 84.0 & 84.0 \\
\hline $\mathrm{o}-\mathrm{H}_{2} \mathrm{CS}$ & thioformaldehyde & $2.0 \times 10^{-10}$ & 8.8 & 6 & $1.1 \times 10^{-10}$ & 58.6 & 209.1 \\
\hline $\mathrm{p}-\mathrm{H}_{2} \mathrm{CS}$ & & $1.8 \times 10^{-10}$ & 2.9 & 4 & $5.7 \times 10^{-11}$ & 46.1 & 98.8 \\
\hline $\mathrm{SiO}$ & silicon monoxide & $5.9 \times 10^{-11}$ & 2.2 & 4 & $1.0 \times 10^{-10}$ & 31.3 & 75.0 \\
\hline${ }^{29} \mathrm{SiO}$ & & $8.8 \times 10^{-12}$ & 5.5 & 3 & $5.0 \times 10^{-12}$ & 31.0 & 74.3 \\
\hline
\end{tabular}

a The reduced $\chi^{2}$ of the best fit model. Based on the $\chi^{2}$-analysis the derived abundances are accurate to about 20-30\% (1 $\left.\sigma\right)$ when the fit is good, i.e., $\chi_{\text {red }}^{2} \sim 1$.

b The number $N$ of independent observational constraints used in the modelling.

${ }^{c}$ Abundance derived by Blake et al. (1994) and van Dishoeck et al. (1995) using a simple excitation analysis.

${ }^{d}$ Lowest energy of the upper level involved in the transitions used as constraints in the modelling.

${ }^{\mathrm{e}}$ Highest energy of the upper level involved in the transitions used as constraints in the modelling.

$\dagger$ Derived from optically thin isotope(s) assuming standard isotopic ratio(s). 
reduces the line optical depths and increases the line intensities thus improving the fit to observations. Material in the outflow can also contribute to these lines.

The observed transitions are only in LTE throughout the envelope for abundant molecules like CO and OCS, including their isotopomers observed here. For less abundant species, where collisional excitation is less efficient, departures from LTE are found. For example, the level populations of common molecules like $\mathrm{CS}$ and $\mathrm{H}_{2} \mathrm{CO}$ are in LTE out to $\sim 1-2 \times 10^{16} \mathrm{~cm}$. For most molecules, populations of the observed lines are in LTE within $\sim 2 \times 10^{15} \mathrm{~cm}$.

From Table 5 it is also evident that the line emission from several molecular species is not fitted well, in particular molecules where the emission probes a large radial range, e.g., in the case of $\mathrm{H}_{2} \mathrm{CO}$ and $\mathrm{CH}_{3} \mathrm{OH}$. In addition, the isotopic ratios derived in many of these cases are far from their interstellar values and what is commonly derived for these kind of objects. Typically, the model intensities from lines sampling the inner parts of the envelope are too low compared to observed values, whereas the opposite is true for the lines probing the outer part of the envelope. An obvious explanation is that a steep gradient is present in the abundances of these molecules.

\subsection{Jump models}

In order to improve the quality of the fits, models with a jump in the molecular abundances are considered. A jump is introduced at the radius in the envelope where the temperature reaches $90 \mathrm{~K}$, at which point the ice starts to evaporate from the grain mantles. In our models, this occurs at $\sim 2 \times 10^{15} \mathrm{~cm}$ or $150 \mathrm{AU}$. The free parameters in the modelling are then the fractional abundances in the inner $\left(f_{\text {in }} ; T>90 \mathrm{~K}\right)$ and outer parts of the envelope $\left(f_{\text {out }} ; T<90 \mathrm{~K}\right)$. For most species, the isotopic abundance ratios are assumed to be fixed to the standard interstellar values to increase the number of constraints used in the modelling. The results from the excitation analysis are presented in Fig. 7 for several species. The reduced $\chi^{2}$ for the best fit models are generally very good, $\sim 1$, and significantly better than the constant abundance models. Typically, a jump of $\sim 100$ in abundance is derived, leading to abundances that are significantly higher than found in quiescent molecular clouds and comparable to those found in the prototypical hot core in Orion (Sect. 6).

The jump models can only be applied to species for which a significant number of lines are observed covering a wide range of excitation conditions. These include $\mathrm{H}_{2} \mathrm{CO}, \mathrm{CH}_{3} \mathrm{OH}$, $\mathrm{CH}_{3} \mathrm{CN}, \mathrm{H}_{2} \mathrm{CS}$, SO and $\mathrm{SO}_{2}$ (see the columns of $E_{\max }$ and $E_{\text {min }}$ included in Table 5). For $\mathrm{HC}_{3} \mathrm{~N}$ and OCS, only lines from highly-excited levels have been observed, so that for these molecules the values of $f_{\text {out }}$ in the outer envelope are poorly constrained. For most simple linear rotors such as HCN, $\mathrm{HCO}^{+}, \mathrm{CN}$, however, the observed lines arise from levels below $90 \mathrm{~K}$, so that no information on the inner warm part is obtained. The only exception is $\mathrm{SiO}$, where the combination of many ${ }^{28} \mathrm{SiO}$ and ${ }^{29} \mathrm{SiO}$ lines allows a jump to be inferred. Molecules such as $\mathrm{HNCO}, \mathrm{CH}_{2} \mathrm{CO}$ and $\mathrm{H}_{2} \mathrm{~S}$ for which only one or two lines are observed and where the emission mainly
Table 6. Various molecular abundances derived using a jump in their fractional abundance, introduced at $T=90 \mathrm{~K}$.

\begin{tabular}{lcccr}
\hline \hline \multicolumn{1}{c}{ Molecule } & $f_{\text {in }}(X)^{\mathrm{a}}$ & $f_{\text {out }}(X)^{\mathrm{b}}$ & $\chi_{\text {red }}^{2}$ & $N$ \\
\hline $\mathrm{HC}_{3} \mathrm{~N}$ & $1.0 \times 10^{-9}$ & $<1.0 \times 10^{-10}$ & 0.1 & 3 \\
$\mathrm{CH}_{3} \mathrm{CN}$ & $7.5 \times 10^{-9}$ & $<8.0 \times 10^{-11}$ & 0.9 & 7 \\
$\mathrm{C}_{3} \mathrm{H}_{2}$ & $<1.5 \times 10^{-9}$ & $1.6 \times 10^{-11}$ & 5.7 & 6 \\
$\mathrm{CH}_{3} \mathrm{C}_{2} \mathrm{H}$ & $3.5 \times 10^{-8}$ & $<1.5 \times 10^{-9}$ & 1.7 & 6 \\
$\mathrm{o}-\mathrm{H}_{2} \mathrm{CO}$ & $4.5 \times 10^{-8}$ & $4.5 \times 10^{-10}$ & 1.9 & 9 \\
$\mathrm{p}^{-} \mathrm{H}_{2} \mathrm{CO}$ & $1.5 \times 10^{-8}$ & $2.5 \times 10^{-10}$ & 1.4 & 8 \\
$\mathrm{H}_{2}^{13} \mathrm{CO}$ & $7.0 \times 10^{-10}$ & $1.0 \times 10^{-11}$ & 1.6 & 6 \\
$\mathrm{HDCO}$ & $2.0 \times 10^{-8}$ & $<9.0 \times 10^{-11}$ & 0.9 & 4 \\
$\mathrm{CH}_{3} \mathrm{OH}$ & $1.0 \times 10^{-7}$ & $3.5 \times 10^{-10}$ & 1.2 & 23 \\
$\mathrm{SO}$ & $2.5 \times 10^{-7}$ & $3.5 \times 10^{-9}$ & 1.8 & 9 \\
$\mathrm{SO}$ & $1.0 \times 10^{-7}$ & $4.5 \times 10^{-10}$ & 0.5 & 10 \\
$\mathrm{OCS}_{\mathrm{o}}$ & $2.5 \times 10^{-7}$ & $<3.0 \times 10^{-9}$ & 1.5 & 7 \\
$\mathrm{p}_{2} \mathrm{CS}$ & $3.0 \times 10^{-9}$ & $1.0 \times 10^{-10}$ & 1.4 & 6 \\
$\mathrm{SiO}$ & $2.5 \times 10^{-9}$ & $<4.0 \times 10^{-11}$ & 0.1 & 4 \\
& $4.5 \times 10^{-9}$ & $2.5 \times 10^{-11}$ & 0.8 & 8 \\
\hline
\end{tabular}

a Abundance in the inner, dense, and hot part of the envelope. ${ }^{b}$ Abundance in the cooler, less dense, outer part of the envelope.

probes hot gas (Table 5) only the inner part of the envelope were modelled. The significantly higher abundances obtained (Sect. 6) compared with the constant abundance models illustrates the point that orders of magnitude higher abundances can be derived if the emission is assumed to originate only from the inner warm region.

The observations analyzed in this paper do not include the lowest rotational transitions probing the coldest outer parts; thus, so-called "anti-jump" models, in which the abundances are decreased below a certain temperature due to freeze-out, cannot be tested, except for the case of $\mathrm{CO}$. There are also some molecules, e.g., $\mathrm{C}_{3} \mathrm{H}_{2}$, for which the jump-models give a worse $\chi^{2}$-fit than the constant abundance models; such molecules are good candidates for the "anti-jump" models if lower transitions are available. In the following, a few individual cases are described in more detail, before discussing the general results.

\section{3. $\mathrm{CO}$}

Carbon monoxide, $\mathrm{CO}$, is difficult to destroy but relatively easy to excite through collisions, even in the outer low-density and cold part of the envelope. Brightness maps of the ${ }^{12} \mathrm{CO}$ molecular line emission associated with IRAS 16293-2422 reveal a complex structure (Walker et al. 1988) indicating two bipolar outflows. Interferometric BIMA observations suggest that the ${ }^{13} \mathrm{CO}(J=1 \rightarrow 0)$ emission is also associated with the outflow to some extent (Schöier et al. 2002a, in prep.). Single-dish observations of higher transitions of ${ }^{13} \mathrm{CO}$ show no direct evidence for tracing the outflow based upon the shape of their line profiles (see Fig. 4). This is also the case for lines from the less abundant $\mathrm{C}^{17} \mathrm{O}$ and $\mathrm{C}^{18} \mathrm{O}$ molecules. Thus these lines can possibly be used to trace the $\mathrm{CO}$ content in the circumstellar envelope.

Assuming a constant abundance throughout the envelope the radiative transfer calculations give abundances of $6.5 \times 10^{-7}$, 

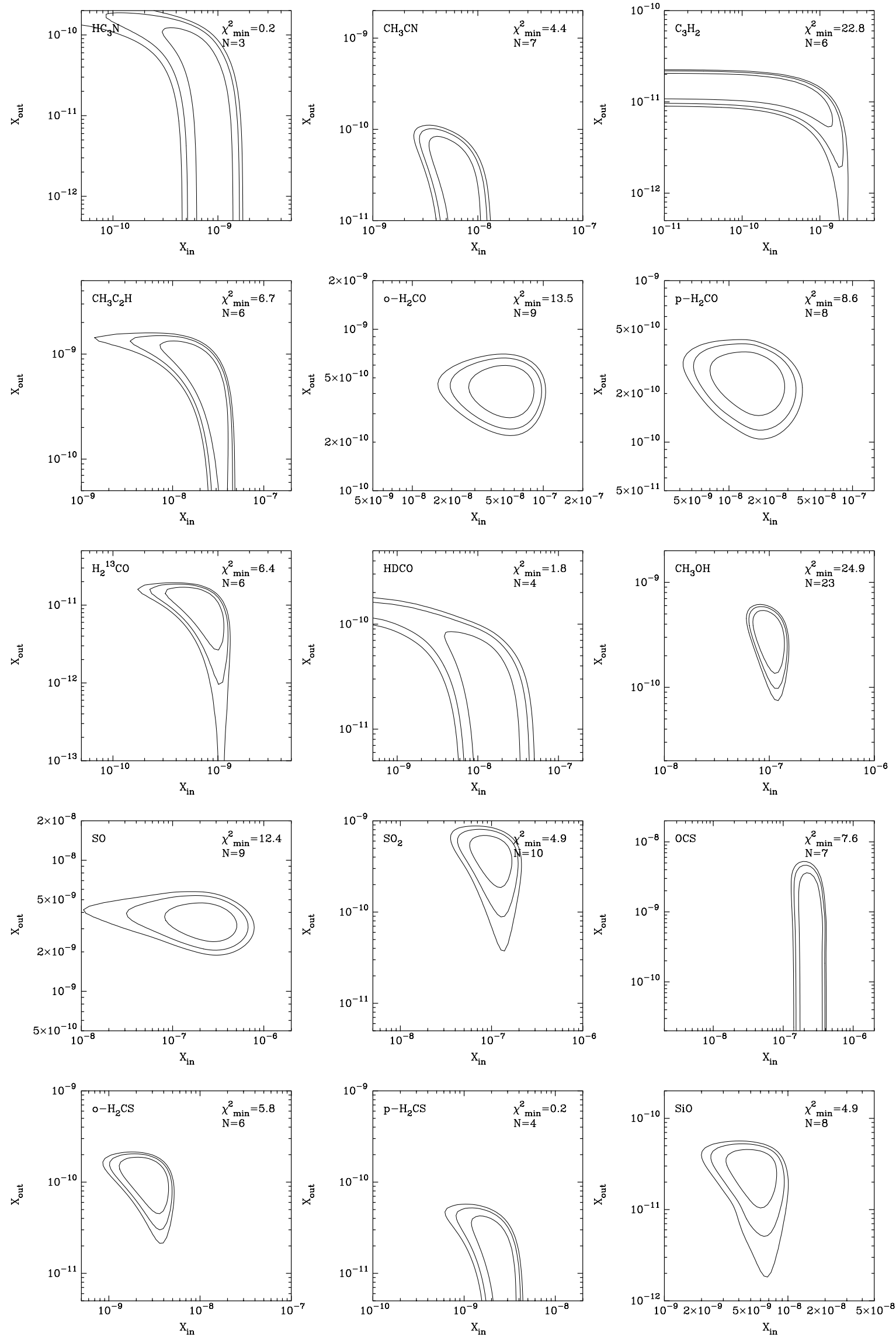

Fig. 7. $\chi^{2}$-maps of various molecular species introducing a step function in abundance at $T_{\text {gas }}=90 \mathrm{~K}$. Contours are drawn at $\chi_{\min }^{2}+(2.3,4.6,6.2)$ indicating the $68 \%, 90 \%$, and $95 \%$ confidence levels, respectively. The number of observational constraints used, $N$, are also shown. The quality of the best fit model can be estimated from the reduced chi-squared $\chi_{\text {red }}^{2}=\chi_{\min }^{2} /(N-2)$. Fixed isotope ratios of ${ }^{28} \mathrm{SiO} /{ }^{29} \mathrm{SiO}=20$, $\mathrm{OCS} / \mathrm{OC}^{34} \mathrm{~S}=20$ and $\mathrm{OCS} / \mathrm{O}^{13} \mathrm{CS}=60$ were assumed. 
$6.2 \times 10^{-8}$ and $1.6 \times 10^{-8}$ for ${ }^{13} \mathrm{CO}, \mathrm{C}^{18} \mathrm{O}$, and $\mathrm{C}^{17} \mathrm{O}$, respectively. The derived $\mathrm{C}^{18} \mathrm{O} / \mathrm{C}^{17} \mathrm{O}$ ratio is 3.9 , in good agreement with typical interstellar values (Wilson \& Rood 1994). Jørgensen et al. (2002) derived ${ }^{18} \mathrm{O} /{ }^{17} \mathrm{O}=3.6 \pm 1.1$ for their survey of protostellar objects. The ${ }^{12} \mathrm{CO}$ abundance is estimated to be $4.0 \times 10^{-5}$ from $\mathrm{C}^{17} \mathrm{O}$ assuming the terrestrial ratio ${ }^{12} \mathrm{CO} / \mathrm{C}^{17} \mathrm{O}=2500$. This is in excellent agreement with the value of $3.9 \times 10^{-5}$ estimated from ${ }^{13} \mathrm{CO}$ using the interstellar value ${ }^{12} \mathrm{CO} /{ }^{13} \mathrm{CO}=60$. In addition, the upper limits obtained for the ${ }^{13} \mathrm{C}^{17} \mathrm{O}(J=2 \rightarrow 1)$ and ${ }^{13} \mathrm{C}^{18} \mathrm{O}(J=3 \rightarrow 2)$ line emission are also consistent with these values. In all, the consistency of the derived values and quality of the model fits (Fig. 4 and Table 5) are reassuring. Due to the high optical depths in the observed ${ }^{13} \mathrm{CO}$ lines, their profiles tend to be somewhat broader than those from the less abundant isotopomers, and their intensities are less sensitive to the assumed abundance. The observed lines are all in LTE, indicating that the derived abundances are not sensitive to the adopted set of collisional rates.

The total inferred $\mathrm{CO}$ abundance is about a factor of two to four lower than the value of $8 \times 10^{-5}$ found in dark clouds (Frerking et al. 1982) and $2 \times 10^{-4}$ in warm regions (Lacy et al. 1994). A plausible explanation is that $\mathrm{CO}$ freezes out in the cool external parts of the envelope. To simulate this situation, a "jump" model with an abrupt decrease in the $\mathrm{CO}$ abundance at $20 \mathrm{~K}$ was introduced; this is a characteristic temperature below which pure-CO ice can exist (Sandford \& Allamandola 1993). To compensate for this freeze-out, the CO abundance in regions above $20 \mathrm{~K}$ needs to be raised. In Fig. 8 the result of varying the $\mathrm{CO}$ abundance in the inner and outer parts of the envelope is presented. A maximum allowed value for the $\mathrm{CO}$ abundance of gas above $20 \mathrm{~K}$ is $\sim 7 \times 10^{-5}$ while at the same time the abundance in the outer cooler envelope needs to be lowered to $\lesssim 2 \times 10^{-5}$, i.e., a depletion of about a factor four. However, in the present analysis a constant abundance model is equally probable. The molecular line modelling performed in Sect. 4.2 suggests that in the envelope around IRAS $16293-2422, T_{\text {gas }} \approx 0.7 \times T_{\text {dust }}$. This in turn means that it is not possible to explain the apparently low $\mathrm{CO}$ abundance with a significant decoupling of the gas temperature from that of the dust.

For the similar modelling of the larger sample of class 0 and I objects, Jørgensen et al. (2002) found that the $\mathrm{CO}$ abundance in general was lower for the class 0 objects than the class I objects (average $\mathrm{CO}$ abundances of respectively $2.0 \times 10^{-5}$ and $\left.1.2 \times 10^{-4}\right)$. Further it was found that abundance jumps at $20 \mathrm{~K}$ of more than a factor 3 could be ruled out in most cases and that constant fractional abundances over the temperature range covered by the $\mathrm{CO}$ rotational lines provided good fits. This lead to the suggestion that $\mathrm{CO}$ in the class 0 objects could be trapped in a porous ice matrix with $\mathrm{H}_{2} \mathrm{O}$ from which it does not fully evaporate until at temperatures of $\sim 60 \mathrm{~K}$. More observational constraints on isotopic $\mathrm{CO}(J=1 \rightarrow 0)$ as well as higher $-J \mathrm{CO}$ lines are needed to verify if $\mathrm{CO}$ is frozen out onto dust grains only at the lowest temperatures or if a substantial fraction of $\mathrm{CO}$ evaporates more gradually up to $\sim 90 \mathrm{~K}$, as suggested by recent experiments of $\mathrm{CO}-\mathrm{H}_{2} \mathrm{O}$ ice mixtures (Collings et al. 2002).

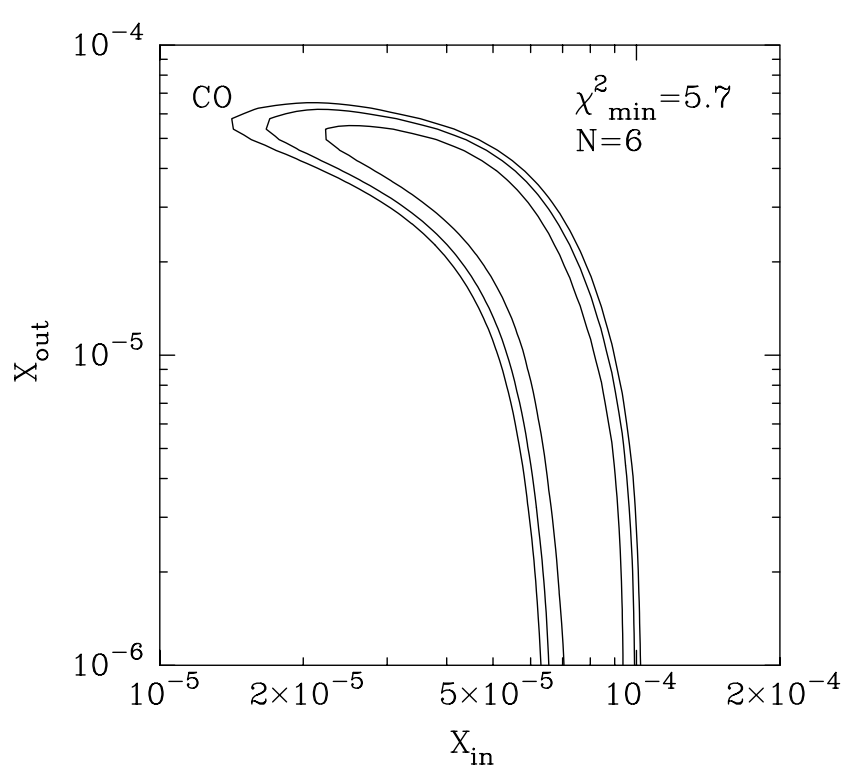

Fig. 8. CO jump-model assuming an abrupt change in abundance at $20 \mathrm{~K}$ using the integrated intensities of the observed ${ }^{13} \mathrm{CO}, \mathrm{C}^{18} \mathrm{O}$, and $\mathrm{C}^{17} \mathrm{O}$ line emission as constraints. Contours are drawn at $\chi_{\min }^{2}+(2.3$, $4.6,6.2)$ indicating the $68 \%, 90 \%$, and $95 \%$ confidence levels, respectively. The number of observational constraints used, $N$, are also shown. The quality of the best fit model can be estimated from the reduced chi-squared $\chi_{\text {red }}^{2}=\chi_{\text {min }}^{2} /(N-2)$.

In Fig. 4 the line profiles obtained from the constant abundance model of the $\mathrm{CO}$ emission are presented. The asymmetry present in some of the observed spectra is not possible to model using a static envelope and requires the presence of a global velocity field, as discussed in Sect. 4.3.

\section{4. $\mathrm{H}_{2} \mathrm{CO}$ and $\mathrm{CH}_{3} \mathrm{OH}$}

$\mathrm{H}_{2} \mathrm{CO}$ and $\mathrm{CH}_{3} \mathrm{OH}$ are two particularly useful molecules to model in detail, because of the relative complexity of the energy level structure of asymmetric rotors and symmetric top molecules. For example, for $\mathrm{H}_{2} \mathrm{CO}$ dipole transitions between various $K_{p}$-ladders are not allowed while collisional transitions are, making these transitions good probes of the temperature structure. In addition, the wealth of available lines in the submillimetre regime makes them ideal to probe various parts of the envelope (Mangum \& Wootten 1993; van Dishoeck et al. 1993; Ceccarelli et al. 2000b). It is clear from Table 6 that the reduced $\chi^{2}$ values are considerably lowered in the jumpmodel for an abundance contrast of $\sim 60-100$ for $\mathrm{H}_{2} \mathrm{CO}$ and $\sim 50$ for $\mathrm{CH}_{3} \mathrm{OH}$. The $\mathrm{H}_{2} \mathrm{CO}$ and $\mathrm{CH}_{3} \mathrm{OH}$ abundances in the cool outer part of the envelope are in line with values in quiescent molecular clouds, whereas those in the inner envelope are of the same order of magnitude as derived for some hot cores (Sect. 6). Typical $\mathrm{CH}_{3} \mathrm{OH}$ and $\mathrm{H}_{2} \mathrm{CO}$ abundances in interstellar ices are $\$ 2 \times 10^{-6}$ and $\sim 10^{-6}$ (Dartois et al. 1999; Keane et al. 2001), illustrating that the drastic increases could be explained by ice evaporation from dust grains above $90 \mathrm{~K}$. For high-mass protostars, van der Tak et al. (2000a) found a similar $\mathrm{CH}_{3} \mathrm{OH}$ abundance jump for some sources, but no evidence for significant jumps in the $\mathrm{H}_{2} \mathrm{CO}$ abundance. The $\mathrm{H}_{2} \mathrm{CO} / \mathrm{CH}_{3} \mathrm{OH}$ ice 
abundance ratio is sensitive to the atomic hydrogen density in the ice-forming region, which could be different for low- and high-mass protostars.

When a significant number of transitions with varying excitation conditions are observed the data have the potential to determine the characteristic temperature at which the majority of the molecules are evaporated. The sensitivity of the data presented here to the adopted jump-temperature can thus be tested for $\mathrm{H}_{2} \mathrm{CO}$ and $\mathrm{CH}_{3} \mathrm{OH}$. The $\mathrm{H}_{2} \mathrm{CO}$ models are not very sensitive to the jump temperature until it drops below about $40 \mathrm{~K}$ at which point the reduced $\chi^{2}$ increases fast. It is found that a temperature of $\sim 50 \mathrm{~K}$ gives the best fit indicating that formaldehyde starts to evaporate at temperatures below $90 \mathrm{~K}$ (see also Ceccarelli et al. 2001). In the case of methanol $\left(\mathrm{CH}_{3} \mathrm{OH}\right)$, the best fit is obtained for $\sim 90 \mathrm{~K}$ whereas temperatures below $\sim 50 \mathrm{~K}$ give poor fits, indicating that this molecule evaporates mainly at $\sim 90 \mathrm{~K}$.

The ortho-to-para ratio of $\mathrm{H}_{2} \mathrm{CO}$ is not well constrained. Based on the $\chi^{2}$-analysis, its lower limit is $\sim 0.9$, with a best-fit value of 2.5. A further complication is that the ortho-to-para ratio may vary, e.g. according to temperature, through the envelope. In the present analysis it is not possible to confirm this. Fixing the ortho-to-para ratio to 2.5 throughout the envelope increases the number of constraints used in the modelling and enables "jump-models" for $\mathrm{H}_{2}{ }^{13} \mathrm{CO}$ and $\mathrm{HDCO}$ to be inferred. From the isotopic ratio, the derived ${ }^{12} \mathrm{C} /{ }^{13} \mathrm{C}$-ratio is $\sim 100$ with considerable uncertainty and fully consistent with the interstellar value of 60 adopted elsewhere in this paper. From deuterated formaldehyde (HDCO) it is possible to estimate the $\mathrm{D} / \mathrm{H}$ ratio and a value $\sim 0.3$ is derived. This is significantly higher than what is obtained from $\mathrm{DCO}^{+} / \mathrm{HCO}^{+}$, $\mathrm{DCN} / \mathrm{HCN}$ and DNC/HNC (see Table 5), and suggests a different scenario for $\mathrm{H}_{2} \mathrm{CO}$. The high degree of deuterium fractionation of $\mathrm{H}_{2} \mathrm{CO}$, further strengthened by the recent detection of $\mathrm{D}_{2} \mathrm{CO}$ in this source (Loinard et al. 2000; Ceccarelli et al. 2001), is about five times larger than values obtained towards other low-mass protostars (Roberts et al. 2002) and dark clouds like TMC-1 and L134N (Turner 2001) but consistent with estimates for the Orion hot core (Turner 1990). Due to the limited number of lines any radial variations in either the ${ }^{12} \mathrm{C} /{ }^{13} \mathrm{C}$-ratio or the deuterium fractionation cannot be established. Ceccarelli et al. (2001) argue, based on spatially resolved emission, that $\mathrm{H}_{2} \mathrm{CO}$ and its deuterated counterparts are formed mainly from grain-surface reactions in a previous cold, dark cloud phase. For $\mathrm{HCO}^{+}, \mathrm{HCN}$ and $\mathrm{HNC}$ the degree of deuteration can be explained by gas-phase reactions at low temperatures (Roberts et al. 2002).

\subsection{Vibrationally excited emission?}

For linear rotors such as CS and HCN, the observed lines cover only a limited range in excitation energy. Much higher frequency data are needed to probe the abundances of these molecules in the warm gas through pure rotational lines in the vibrational ground state (e.g., Boonman et al. 2001). Some lines of vibrationally-excited molecules have been detected toward IRAS 16293-2422, however, in particular the CS $(v=1$;
$J=7 \rightarrow 6$ ) line. Since this line originates from a level at about $1900 \mathrm{~K}$ above ground, it cannot be excited by collisions only. Instead, radiative excitation by dust emission must play a role.

The addition of radiative excitation by dust is straightforward in the Monte Carlo scheme if scattering is assumed to be negligible, which is the case at the wavelengths of importance here: the CS fundamental vibrational transition occurs around $8 \mu \mathrm{m}$. In the absence of scattering events only emission and absorption by the dust particles need to be considered. The dust is assumed to locally emit thermal radiation described by the dust temperature $T(r)$, according to Kirchhoff's law. The model photons emitted by the dust are released together with the other model photons and the additional opacity provided by the dust is added to the line optical depth.

The CS abundance of $2.5 \times 10^{-9}$ derived previously fails to account for the observed CS $(v=1 ; J=7 \rightarrow 6)$ line emission by many orders of magnitudes. Even a "jump-model" with the abundance increased by a factor of 100 fails to account for all the observed emission.

Recently, Highberger et al. (2000) failed to detect any vibrationally excited CS emission in lower $J$-transitions towards IRAS $16293-2422$ down to $T_{\text {rms }} \sim 10 \mathrm{mK}$. One possibility is that the $\operatorname{CS}(v=1 ; J=7 \rightarrow 6)$ line was mis-identified by Blake et al. (1994). Their observations were obtained in dual sideband and the CS line was supposed to reside in the lower sideband. However, in the upper sideband two $\mathrm{H}_{2} \mathrm{CS}$ lines $\left(J_{K_{-}, K_{+}}=10_{5,6} \rightarrow 9_{5,5}\right.$ and $\left.J_{K_{-}, K_{+}}=10_{5,5} \rightarrow 9_{5,4}\right)$ at $343.202331 \mathrm{GHz}$ coincide with the position of the CS line in the lower sideband. To check the possibility that these transitions contribute significantly to the observed intensity of the line, a LTE jump-model was run using the parameters derived previously for $\mathrm{H}_{2} \mathrm{CS}$. It is indeed found that these high lying energy levels $\left(E_{\mathrm{u}}=418 \mathrm{~K}\right)$ are sufficiently excited to account for all of the flux observed in this line.

\section{Discussion}

\subsection{Does IRAS 16293-2422 have a hot core?}

Hot cores have been defined for high-mass sources as small $(<0.1 \mathrm{pc})$, dense $\left(n_{\mathrm{H}_{2}}>10^{7} \mathrm{~cm}^{-3}\right)$ and warm $(T>100 \mathrm{~K})$ regions (Walmsley 1992; Kurtz et al. 2000). The chemical signatures of hot cores are high abundances of a wide variety of complex organic molecules and fully hydrogenated molecules such as $\mathrm{H}_{2} \mathrm{O}, \mathrm{NH}_{3}$ and $\mathrm{H}_{2} \mathrm{~S}$. These chemical characteristics are thought to arise from thermal evaporation of ice mantles from the grains close to the protostar, followed by rapid hightemperature gas-phase reactions for a period of $10^{4}-10^{5} \mathrm{yr}$ (Charnley et al. 1992). In this scenario, the fully hydrogenated species are "first generation" molecules produced by surface chemistry on the grains, whereas complex organic molecules like $\mathrm{HC}_{3} \mathrm{~N}, \mathrm{CH}_{3} \mathrm{OCH}_{3}$ and $\mathrm{HCOOCH}_{3}$ are "second generation" produced by gas-phase chemistry between evaporated species. To what extent do these physical and chemical characteristics also apply to the low-mass object IRAS 16293-2422?

The dust radiative transfer modelling performed in Sect. 4 clearly indicates that there is evidence for the presence of hot and dense material within $\sim 150 \mathrm{AU}$ of the protostar. Moreover, 
Table 7. Summary of derived abundances in the envelope of IRAS 16293-2422.

\begin{tabular}{|c|c|c|c|c|c|c|c|}
\hline Molecule & $f_{\text {in }}(X)^{\mathrm{a}}$ & $f_{\text {out }}(X)^{\mathrm{b}}$ & $\mathrm{L} 134 \mathrm{~N}^{\mathrm{c}}$ & $\begin{array}{l}\text { Orion }^{\mathrm{d}} \\
\text { hot core }\end{array}$ & $\begin{array}{l}\text { L1157 } \\
\text { outflow }\end{array}$ & Ices $^{f}$ & $\begin{array}{c}\text { Comets }^{\mathrm{g}} \\
\text { Hale - Bopp }\end{array}$ \\
\hline $\mathrm{CO}$ & $\ldots$ & $4.0 \times 10^{-5}$ & $1 \times 10^{-4}$ & $1 \times 10^{-4}$ & $1 \times 10^{-4}$ & $(1-5) \times 10^{-6}$ & $1 \times 10^{-5}$ \\
\hline $\mathrm{HCO}^{+}$ & $\ldots$ & $1.4 \times 10^{-9}$ & $8 \times 10^{-9}$ & $1 \times 10^{-9}$ & $3 \times 10^{-8}$ & $\cdots$ & $\ldots$ \\
\hline $\mathrm{CN}$ & $\cdots$ & $8.0 \times 10^{-11}$ & $5 \times 10^{-10}$ & $\cdots$ & $5 \times 10^{-8}$ & $\cdots$ & $\cdots$ \\
\hline $\mathrm{HCN}$ & $\ldots$ & $1.1 \times 10^{-9}$ & $7 \times 10^{-9}$ & $4 \times 10^{-7}$ & $5 \times 10^{-7}$ & $<2 \times 10^{-6}$ & $1 \times 10^{-7}$ \\
\hline $\mathrm{HNC}$ & $\ldots$ & $6.9 \times 10^{-11}$ & $3 \times 10^{-8}$ & $\ldots$ & $5 \times 10^{-8}$ & $\ldots$ & $2 \times 10^{-8}$ \\
\hline $\mathrm{HC}_{3} \mathrm{~N}$ & $1.0 \times 10^{-9}$ & $<1.0 \times 10^{-10}$ & $4 \times 10^{-10}$ & $2 \times 10^{-9}$ & $1 \times 10^{-8}$ & $\ldots$ & $1 \times 10^{-8}$ \\
\hline $\mathrm{CH}_{3} \mathrm{CN}$ & $7.5 \times 10^{-9}$ & $<8.0 \times 10^{-11}$ & $<4 \times 10^{-10}$ & $2 \times 10^{-8}$ & $\ldots$ & $\cdots$ & $1 \times 10^{-8}$ \\
\hline $\mathrm{HNCO}$ & $9.0 \times 10^{-9^{\mathrm{h}}}$ & $\ldots$ & $\ldots$ & $6 \times 10^{-9}$ & $\ldots$ & $\sim 2 \times 10^{-6}$ & $5 \times 10^{-8}$ \\
\hline $\mathrm{C}_{2} \mathrm{H}$ & $\ldots$ & $2.2 \times 10^{-10}$ & $2 \times 10^{-9}$ & $\cdots$ & $\cdots$ & $\ldots$ & $\ldots$ \\
\hline $\mathrm{C}_{3} \mathrm{H}_{2}$ & $\ldots$ & $1.6 \times 10^{-11}$ & $2 \times 10^{-9}$ & $\ldots$ & $\cdots$ & $\cdots$ & $\ldots$ \\
\hline $\mathrm{CH}_{3} \mathrm{C}_{2} \mathrm{H}$ & $3.5 \times 10^{-8}$ & $<1.5 \times 10^{-9}$ & $<1 \times 10^{-9}$ & $1 \times 10^{-9}$ : & $\ldots$ & $\ldots$ & $\ldots$ \\
\hline $\mathrm{H}_{2} \mathrm{CO}$ & $6.0 \times 10^{-8}$ & $7.0 \times 10^{-10}$ & $2 \times 10^{-8}$ & $1 \times 10^{-8}$ & $3 \times 10^{-7}$ & $(1-4) \times 10^{-6}$ & $5 \times 10^{-7}$ \\
\hline $\mathrm{CH}_{3} \mathrm{OH}$ & $3.0 \times 10^{-7}$ & $3.5 \times 10^{-10}$ & $5 \times 10^{-9}$ & $2 \times 10^{-7}$ & $2 \times 10^{-5}$ & $(2-10) \times 10^{-6}$ & $1 \times 10^{-6}$ \\
\hline $\mathrm{CH}_{2} \mathrm{CO}$ & $5.0 \times 10^{-8^{\mathrm{h}}}$ & $\ldots$ & $<7 \times 10^{-10}$ & $3 \times 10^{-10}$ & $\ldots$ & $\ldots$ & $\ldots$ \\
\hline $\mathrm{HCOOH}$ & $<8.0 \times 10^{-9^{\mathrm{h}}}$ & $\ldots$ & $3 \times 10^{-10}$ & $8 \times 10^{-10}$ & $\ldots$ & $(2-10) \times 10^{-7}$ & $5 \times 10^{-8}$ \\
\hline $\mathrm{CH}_{3} \mathrm{CHO}$ & $<2.0 \times 10^{-9 \mathrm{~h}}$ & $\cdots$ & $6 \times 10^{-10}$ & $\lesssim 2 \times 10^{-10}$ & $\cdots$ & $\cdots$ & $\cdots$ \\
\hline $\mathrm{CH}_{3} \mathrm{OCH}_{3}$ & $<4.0 \times 10^{-8^{\mathrm{h}}}$ & $\ldots$ & $\ldots$ & $1 \times 10^{-8}$ & $\ldots$ & $\ldots$ & $\ldots$ \\
\hline $\mathrm{HCOOCH}_{3}$ & $<6.0 \times 10^{-8^{h}}$ & $\cdots$ & $\cdots$ & $1 \times 10^{-8}$ & $\cdots$ & $\cdots$ & $4 \times 10^{-6}$ \\
\hline $\mathrm{CS}$ & $\ldots$ & $3.0 \times 10^{-9}$ & $1 \times 10^{-9}$ & $1 \times 10^{-8}$ & $2 \times 10^{-7}$ & $\ldots$ & $\ldots$ \\
\hline SO & $2.5 \times 10^{-7}$ & $3.5 \times 10^{-9}$ & $6 \times 10^{-9}$ & $5 \times 10^{-8}$ & $3 \times 10^{-7}$ & $\cdots$ & $1 \times 10^{-7}$ \\
\hline $\mathrm{SO}_{2}$ & $1.0 \times 10^{-7}$ & $4.5 \times 10^{-10}$ & $3 \times 10^{-9}$ & $6 \times 10^{-8}$ & $5 \times 10^{-7}$ & $\cdots$ & $1 \times 10^{-7}$ \\
\hline OCS & $2.5 \times 10^{-7}$ & $<3.0 \times 10^{-9}$ & $7 \times 10^{-9}$ & $5 \times 10^{-8}$ & $2 \times 10^{-7}$ & $2 \times 10^{-7}$ & $2 \times 10^{-7}$ \\
\hline $\mathrm{HCS}^{+}$ & $\cdots$ & $2.4 \times 10^{-11}$ & $1 \times 10^{-10}$ & $\cdots$ & $3 \times 10^{-9}$ & $\cdots$ & $\cdots$ \\
\hline $\mathrm{H}_{2} \mathrm{CS}$ & $5.5 \times 10^{-9}$ & $1.0 \times 10^{-10}$ & $\ldots$ & $\ldots$ & $\ldots$ & $\ldots$ & $1 \times 10^{-8}$ \\
\hline $\mathrm{H}_{2} \mathrm{~S}$ & $9.0 \times 10^{-8}$ & $\cdots$ & $8 \times 10^{-10}$ & $<1 \times 10^{-7}$ & $4 \times 10^{-7}$ & $\cdots$ & $7 \times 10^{-7}$ \\
\hline $\mathrm{SiO}$ & $4.5 \times 10^{-9}$ & $2.5 \times 10^{-11}$ & $<4 \times 10^{-12}$ & $6 \times 10^{-8}$ & $7 \times 10^{-8}$ & $\ldots$ & $\ldots$ \\
\hline
\end{tabular}

a Abundance in warm and dense inner part of the envelope $\leq 150$ AU in radius around IRAS 16293-2422.

${ }^{b}$ Abundance in cooler, less dense outer part of the envelope around IRAS 16293-2422.

${ }^{\mathrm{c}}$ From Ohishi et al. (1992), updated using values from (2000) for L134N pos. C .

${ }^{\mathrm{d}}$ From van Dishoeck \& Blake (1998) (and references cited); otherwise from Sutton et al. (1995).

${ }^{\text {e }}$ From Bachiller \& Pérez Gutiérrez (1997) at position B2 assuming $\mathrm{CO} / \mathrm{H}_{2}=10^{-4}$.

${ }^{\mathrm{f}}$ Based on Ehrenfreund \& Charnley (2000) assuming $\mathrm{H}_{2} \mathrm{O}$ (ice) $/ \mathrm{H}_{2}=5 \times 10^{-5}$. The ranges reflect the values found for different sources. The value for $\mathrm{HNCO}$ is derived from $\mathrm{OCN}^{-}$, assuming this ice evaporates as $\mathrm{HNCO}$.

${ }^{g}$ From Bockelée-Morvan et al. (2000) for comet Hale-Bopp at $1 \mathrm{AU}$, assuming $\mathrm{H}_{2} \mathrm{O} / \mathrm{H}_{2}=5 \times 10^{-5}$.

${ }^{\mathrm{h}}$ Assuming that the observed emission or upper limits apply to the inner warm region only.

this material is most probably in a state of collapse towards the protostar. The need for introducing drastic jumps in the abundances of some "first generation" species like $\mathrm{H}_{2} \mathrm{CO}$ and $\mathrm{CH}_{3} \mathrm{OH}$ at the evaporation temperature of the ices $(\sim 90 \mathrm{~K})$ further suggests that the ice mantles are liberated in the hot inner regions. Table 7 compares the abundances $f_{\text {in }}$ of these molecules with those found in interstellar ices and in highmass hot cores. The table also includes the abundances found in comet Hale-Bopp, which may be representative of interstellar ices. Although the IRAS 16293-2422 abundances are still up to an order of magnitude lower than those found in typical ices, they are comparable to those observed in high-mass hot cores. The high degree of deuterium fractionation measured for these molecules (van Dishoeck et al. 1995; Ceccarelli et al. 2001) is consistent with this interpretation, and probably results from a combination of gas-phase deuterium fractionation in the cold pre-collapsing cloud and grain-surface reactions (Tielens 1983; Roberts \& Millar 2000).

The high abundances of the sulfur-bearing species of $\sim 10^{-7}$ may also fit with this scenario. Molecules like $\mathrm{SO}, \mathrm{SO}_{2}, \mathrm{OCS}$ and $\mathrm{H}_{2} \mathrm{CS}$ are all predicted to be drastically enhanced by the injection of $\mathrm{H}_{2} \mathrm{~S}$ into the hot core region (Charnley 1997), but may also be present in the grain mantles themselves. $\mathrm{H}_{2} \mathrm{~S}$ is detected toward IRAS 16293-2422, but because only a single line is observed, the jump model cannot be uniquely constrained. If all $\mathrm{H}_{2} \mathrm{~S}$ emission is assumed to come from the hot inner region, 
its inferred abundance is $\sim 1 \times 10^{-7}$. This relatively high $\mathrm{H}_{2} \mathrm{~S}$ abundance is consistent with values found in a survey of massive hot cores (Hatchell et al. 1998) but an order of magnitude lower than the estimates for Orion (Minh et al. 1990).

The physical characteristics in IRAS 16293-2422 are thus consistent with those of a typical "hot core" as observed towards many high mass protostars, except for a large change in physical scale. The remaining question is whether the evaporated ices have driven a similarly complex organic chemistry in this low-mass protostar. Among potential "second generation" products, $\mathrm{HC}_{3} \mathrm{~N}$ and $\mathrm{CH}_{3} \mathrm{CN}$ are observed in fairly large amounts with some evidence for jumps in their abundances. However, the IRAS 16293-2422 spectra do not show the wealth of spectral features due to other complex organics - although the confusion limit is clearly not yet reached. The limits on the abundances of molecules such as $\mathrm{CH}_{3} \mathrm{OCH}_{3}$ and $\mathrm{HCOOCH}_{3}$, derived if it is assumed that these molecules are located only in the warm inner part of the envelope, are higher than those found for high-mass protostars (Table 7). Deep integrations down to $\sim 5-10 \mathrm{mK}$ are needed to verify the presence of these complex organic species.

Typically, species such as $\mathrm{SO}_{2}, \mathrm{OCS}, \mathrm{HC}_{3} \mathrm{~N}$ and $\mathrm{CH}_{3} \mathrm{CN}$ are assumed to be "second generation" products, in which case their abundance ratios can be used as "chemical clocks" to determine the time since the evaporation of the ice mantles. For the density and temperature prevailing in the inner parts of the envelope around IRAS 16293-2422 an age of $\sim 10^{4} \mathrm{yr}$ is inferred from such chemical models, a value that is rather uncertain and sensitive to the cosmic ray ionization rate (e.g., Charnley 1997; Hatchell et al. 1998). However, if the velocity field derived from the dust modelling for the Shu infall model is correct, the transit time for grains and molecules through the warm, dense region surrounding IRAS $16293-2422$ is only several hundred years. This is not sufficient time for extensive second generation processing to result, and would naturally explain the lack of large, complex organic species toward this source if their abundances are indeed found to be very low by subsequent deep searches. In such a scenario, the observed abundances would provide a unique opportunity to trace the chemical richness derived from previous stages of grain mantle and gas phase chemistry at sensitivities much higher than those achieved via infrared spectroscopy of icy grains.

\subsection{Alternative scenarios}

The above discussion demonstrates that the IRAS 16293-2422 data can be consistently interpreted with a "hot core" in which the ices are liberated from the grains by thermal evaporation above $90 \mathrm{~K}$. However, one problem is that the size of the "hot core" is only $\sim 150 \mathrm{AU}$ in radius, comparable to the size(s) of the circumstellar disk(s), and much smaller than the binary separation. On this scale, departures from spherical symmetry can be expected to play a role and the disk can shadow part of the inner envelope, keeping it much colder. Moreover, the IRAS 16293-2422 envelope is known to be traversed by outflows. To what extent could these processes affect the interpretation? The data clearly indicate jumps in the abundances of

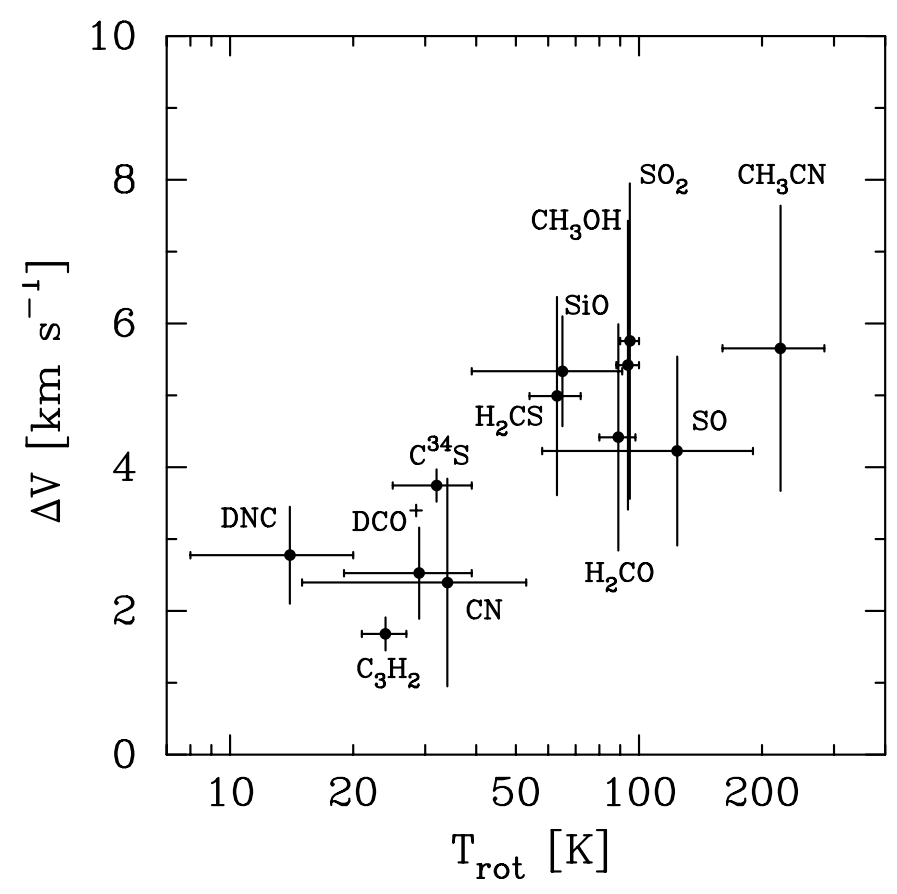

Fig. 9. Observed line widths $(\Delta V)$ of different molecules as functions of their estimated rotational excitation temperature $\left(T_{\text {rot }}\right)$.

"first generation" molecules known to be present in interstellar ices, but could these icy mantles also be removed by alternative mechanisms?

Blake et al. (1994) and van Dishoeck et al. (1995) proposed that grain-grain collisions in the turbulent shear zones where the outflow interacts with the envelope can also be effective. This mechanism is observed for the class 0 protostar L1157, where the outflow interaction can be spatially separated from the immediate protostellar environment with single-dish telescopes. The inferred abundances by Bachiller \& Pérez Gutiérrez (1997) are included in Table 7 and are seen to also be close to the values found in the inner region of IRAS 16293-2422, especially for the sulfur-bearing species.

An interpretation in which the ices are liberated by mild shocks or turbulence rather than thermal evaporation has two observational consequences. First, the lines of the "first generation" ice mantle species are expected to be wider and have a different velocity structure than those of molecules located predominantly in the quiescent outer envelope. Figure 9 shows the observed line widths of different molecules as functions of their excitation temperature, where the values are taken from Blake et al. (1994) and van Dishoeck et al. (1995). Molecules with clear "jumps" in their abundances $\left(\mathrm{SO}_{2}, \mathrm{CH}_{3} \mathrm{OH}, \mathrm{CH}_{3} \mathrm{CN}\right)$ have larger line widths and higher excitation temperatures than molecules which trace the outer envelope $\left(\mathrm{CN}, \mathrm{C}_{2} \mathrm{H}, \mathrm{DNC}\right.$, $\mathrm{DCO}^{+}$). The line widths observed towards IRAS 16293-2422 are significantly wider than what is typically observed for class 0 sources $\left(\lesssim 1 \mathrm{~km} \mathrm{~s}^{-1}\right.$ for $\mathrm{C}^{18} \mathrm{O}$ and $\mathrm{C}^{17} \mathrm{O}$; Jørgensen et al. 2002) The observed widths up to $8 \mathrm{~km} \mathrm{~s}^{-1}$ could, however, possibly be explained by the infall model with thermal evaporation (Fig. 5) and are not clear-cut evidence for association with the molecular outflow, illustrating the difficulty in 
disentangling the contributions from various velocity components. Second, the spatial distribution of the "first generation" molecules will be different. Molecules produced by thermal evaporation should be located within a $\sim 150 \mathrm{AU}\left(1^{\prime \prime}\right)$ radius, whereas in the shock scenario they are expected to coat the walls of the outflow(s). Such an "X-type" geometry can extend over a much larger region, even though the total mass of warm gas may be comparable to that in the first model. Chemically, there is expected to be little difference between the two scenarios, except perhaps in the "second generation" products if the liberation by shocks occurs in lower density or temperature gas. Observations at sub-arcsec resolution with the Smithsonian SubMillimeter Array (SMA) and the Atacama Large Millimeter Array (ALMA) are needed to distinguish these scenarios.

Recently, Viti et al. (2001) have suggested, based on chemical modelling, that some molecular abundance ratios are strongly affected by the presence of a shock. In these models, the shocks not only liberate the ice mantles but also drive high-temperature $(\sim 2000 \mathrm{~K})$ reactions. In particular the combination of $\mathrm{HCO} / \mathrm{H}_{2} \mathrm{CO}$ and $\mathrm{NS} / \mathrm{CS}$ ratios may be suited for tracing the dynamical history of a hot core. For the $\mathrm{HCO} / \mathrm{H}_{2} \mathrm{CO}$ ratio we derive an upper limit of $\sim 1$, about an order of magnitude larger than any model predictions, whereas no NS data are available. Thus, the abundances derived from the data set cannot be used to constrain their origin in the inner hot region of IRAS 16293-2422.

\section{Conclusions}

The continuum emission radiated by dust grains in the circumstellar environment of recently born stars can be used, when supplemented by a detailed radiative transfer analysis, to derive the physical properties of such dusty envelopes. Observational constraints in the form of both the SED and resolved radial brightness distributions are required for a successful model. For the protostellar object IRAS 16293-2422 it is possible to model the observational data using a single power-law distribution for the density structure. However, the molecular line profiles suggest that parts of the envelope are in a state of collapse. A dynamical age of $\sim 1-3 \times 10^{4} \mathrm{yr}$ is derived with an annual mass accretion rate of roughly $4 \times 10^{-5} M_{\odot} \mathrm{yr}^{-1}$.

Once the physical structure of IRAS 16293-2422 is known, it is possible to determine its chemical properties through detailed radiative transfer modelling of the observed molecular line emission. The abundances and quality of the fits for molecules like CO and CS further strengthen the adopted physical model. While the emission from some molecules is well reproduced assuming a constant fractional abundance throughout the envelope, other species require a steep abundance gradient to be introduced at typically $90 \mathrm{~K}$. The presence of such a jump for molecules like $\mathrm{H}_{2} \mathrm{CO}$ and $\mathrm{CH}_{3} \mathrm{OH}$ is interpreted as evidence of thermal evaporation of ices in the inner dense and hot regions of the envelope. Other molecules like $\mathrm{HC}_{3} \mathrm{~N}$, $\mathrm{CH}_{3} \mathrm{CN}$ and several sulfur-bearing molecules also require such drastic jumps in their abundance distributions. In high mass protostars, these molecules are typically cited as evidence of a rapid gas-phase chemistry initiated by the evaporation of the ices. The "hot core" region in IRAS 16293-2422 is, however, very small, only $\sim 150 \mathrm{AU}$ in radius and comparable to the size of the circumstellar disk(s). This large change in physical size and the organized velocity field surrounding low mass protostars lead to chemical time scales that only a small fraction of the dynamical age. Thus, it may be difficult for a full "hot core" chemistry leading to complex species such as $\mathrm{CH}_{3} \mathrm{OCH}_{3}$ to develop in class 0 objects, even though the appropriate physical conditions are present. Alternative scenarios in which ices are liberated by grain-grain collisions in turbulent shear zones associated with the outflows need to be tested by higher angular resolution observations.

Whatever their precise origin, the molecules located in the inner envelope of IRAS 16293-2422 can be incorporated into the growing circumstellar disk(s) and become part of the material from which planetary bodies are formed. The molecular abundances derived here should provide an accurate reference point for comparison with the growing amount of data on protoplanetary disks and icy solar system objects such as comets.

Acknowledgements. The authors are grateful to R. Stark and S. Doty for useful discussions. The referee J. Hatchell is thanked for comments that helped to improve the paper. This research was supported by the Netherlands Organization for Scientific Research (NWO) grant 614.041.004, the Netherlands Research School for Astronomy (NOVA) and a NWO Spinoza grant. This article made use of data obtained through the JCMT archive as Guest User at the Canadian Astronomy Data Center, which is operated by the Dominion Astrophysical Observatory for the National Research Council of Canada's Herzberg Institute of Astrophysics.

\section{References}

André, P., Ward-Thompson, D., \& Barsony, M. 1993, ApJ, 406, 122

André, P., Ward-Thompson, D., \& Barsony, M. 2000, in Protostars and Planets IV, ed. V. Mannings, A. Boss, \& S. Russell (Tucson: Univ. Arizona Press), 59

Archibald, E. N., Wagg, J. W., \& Jenness, T. 2000, Calculating Sky Opacities: a re-analysis for SCUBA data (SCD System note 1.1)

Bachiller, R., \& Pérez Gutiérrez, M. 1997, ApJ, 487, L93

Blake, G. A., van Dishoeck, E. F., Jansen, D. J., Groesbeck, T. D., \& Mundy, L. G. 1994, ApJ, 428, 680

Bockelée-Morvan, D., Lis, D. C., Wink, J. E., et al. 2000, A\&A, 353, 1101

Boonman, A. M. S., Stark, R., van der Tak, F. F. S., et al. 2001, ApJ, 553, L63

Castets, A., Ceccarelli, C., Loinard, L., Caux, E., \& Lefloch, B. 2001, A\&A, 375, 40

Ceccarelli, C., Castets, A., Caux, E., et al. 2000a, A\&A, 355, 1129

Ceccarelli, C., Hollenbach, D. J., \& Tielens, A. G. G. M. 1996, ApJ, 471,400

Ceccarelli, C., Loinard, L., Castets, A., Tielens, A. G. G. M., \& Caux, E. 2000b, A\&A, 357, L9

Ceccarelli, C., Loinard, L., Castets, A., et al. 2001, A\&A, 372, 998

Chandler, C. J., \& Richer, J. S. 2000, ApJ, 530, 851

Charnley, S. B. 1997, ApJ, 481, 396

Charnley, S. B., Kress, M. E., Tielens, A. G. G. M., \& Millar, T. J. 1995, ApJ, 448, 232 
Charnley, S. B., Tielens, A. G. G. M., \& Millar, T. J. 1992, ApJ, 399, L71

Collings, M. P., Dever, J. W., Fraser, H. J., McCoustra, M. R. S., \& Williams, D. A. 2002, ApJ, accepted [astro-ph/0205068]

Dartois, E., Schutte, W., Geballe, T. R., et al. 1999, A\&A, 342, L32

Dickens, J. E., Irvine, W. M., Snell, R. L., et al. 2000, ApJ, 542, 870

Doty, S. D., \& Leung, C. M. 1994, ApJ, 424, 729

Doty, S. D., \& Neufeld, D. A. 1997, ApJ, 489, 122

Doty, S. D., \& Palotti, M. L. 2002, MNRAS, in press

Ehrenfreund, P., \& Charnley, S. B. 2000, ARA\&A, 38, 427

Evans, N. J. 1999, ARA\&A, 37, 311

Frerking, M. A., Langer, W. D., \& Wilson, R. W. 1982, ApJ, 262, 590

Hatchell, J., Fuller, G. A., Millar, T. J., Thompson, M. A., \& Macdonald, G. H. 2000, A\&A, 357, 637

Hatchell, J., Thompson, M. A., Millar, T. J., \& MacDonald, G. H. 1998, A\&A, 338, 713

Highberger, J. L., Apponi, A. J., Bieging, J. H., Ziurys, L. M., \& Mangum, J. G. 2000, ApJ, 544, 881

Hogerheijde, M. R., \& Sandell, G. 2000, ApJ, 534, 880

Hogerheijde, M. R., \& van der Tak, F. F. S. 2000, A\&A, 362, 697

Holland, W. S., Robson, E. I., Gear, W. K., et al. 1999, MNRAS, 303, 659

Ivezić, Ž., \& Elitzur, M. 1997, MNRAS, 287, 799

Ivezić, Ž., Nenkova, M., \& Elitzur, M. 1999, User Manual for DUSTY (Univ. Kentucky Internal Rep.)

Jenness, T., \& Lightfoot, J. F. 1997, Starlink User Note 216 (see also the SURF homepage: http://www.jch.hawaii.edu/jcmt_sw/scuba/surf/)

Jørgensen, J. K., Schöier, F. L., \& van Dishoeck, E. F. 2002, A\&A, accepted [astro-ph/0205068]

Keane, J. V., Tielens, A. G. G. M., Boogert, A. C. A., Schutte, W. A., \& Whittet, D. C. B. 2001, A\&A, 376, 254

Kuan, Y., \& Snyder, L. E. 1996, ApJ, 470, 981

Kurtz, S., Cesaroni, R., Churchwell, E., Hofner, P., \& Walmsley, C. M. 2000, in Protostars and Planets IV, ed. V. Mannings, A. Boss, \& S. Russell (Tucson: Univ. Arizona Press), 299

Lacy, J. H., Knacke, R., Geballe, T. R., \& Tokunaga, A. T. 1994, ApJ, 428, L69

Langer, W. D., van Dishoeck, E. F., Bergin, E. A., et al. 2000, in Protostars and Planets IV, ed. V. Mannings, A. Boss, \& S. Russell (Tucson: Univ. Arizona), 29

Loinard, L., Castets, A., Ceccarelli, C., et al. 2000, A\&A, 359, 1169

Looney, L. W., Mundy, L. G., \& Welch, W. J. 2000, ApJ, 529, 477

Mangum, J. G., \& Wootten, A. 1993, ApJS, 89, 123

Menten, K. M., Serabyn, E., Guesten, R., \& Wilson, T. L. 1987, A\&A, 177, L57

Millar, T. J., MacDonald, G. H., \& Gibb, A. G. 1997, A\&A, 325, 1163

Minh, Y. C., Irvine, W. M., McGonagle, D., \& Ziurys, L. M. 1990, ApJ, 360, 136

Mizuno, A., Fukui, Y., Iwata, T., Nozawa, S., \& Takano, T. 1990, ApJ, 356,184

Motte, F., \& André, P. 2001, A\&A, 365, 440

Mundy, L. G., Wilking, B. A., \& Myers, S. T. 1986, ApJ, 311, L75

Mundy, L. G., Wootten, A., Wilking, B. A., Blake, G. A., \& Sargent, A. I. 1992, ApJ, 385, 306

Mundy, L. G., Wootten, H. A., \& Wilking, B. A. 1990, ApJ, 352, 159

Myers, P. C., Evans, N. J., \& Ohashi, N. 2000, in Protostars and Planets IV, ed. V. Mannings, A. Boss, \& S. Russell (Tucson: Univ. Arizona Press), 217
Narayanan, G., Walker, C. K., \& Buckley, H. D. 1998, ApJ, 496, 292

Ohishi, M., Irvine, W. M., \& Kaifu, N. 1992, in Astrochemistry of Cosmic Phenomena, ed. P. D. Singh (Kluwer: Dordrecht), IAU Symp., 150, 171

Ossenkopf, V., \& Henning, T. 1994, A\&A, 291, 943

Roberts, H., Fuller, G. A., Millar, T. J., Hatchell, J., \& Buckle, J. V. 2002, A\&A, 381, 1026

Roberts, H., \& Millar, T. J. 2000, A\&A, 364, 780

Rodgers, S. D., \& Charnley, S. B. 2001, ApJ, 546, 324

Sandell, G. 1997, The SCUBA mapping cookbook (Starlink Cookbook 11.1)

Sandford, S. A., \& Allamandola, L. J. 1993, Icarus, 106, 478

Schöier, F. L. 2000, Ph.D. Thesis, Stockholm Observatory, SE-133 36 Saltsjöbaden, Sweden

Schöier, F. L., \& Olofsson, H. 2001, A\&A, 368, 969

Schilke, P. 2000, in Astrochemistry: From Molecular Clouds to Planetary Systems, ed. Y. C. Minh, \& E. F. van Dishoeck (ASP), 575

Shirley, Y. L., Evans, N. J., Rawlings, J. M. C., \& Gregersen, E. M. 2000, ApJS, 131, 249

Shu, F., Najita, J., Galli, D., Ostriker, E., \& Lizano, S. 1993, in Protostars and Planets III, ed. E. H. Levy, \& J. Lunine (Tucson: Univ. Arizona Press), 3

Shu, F. H. 1977, ApJ, 214, 488

Shu, F. H., Adams, F. C., \& Lizano, S. 1987, ARA\&A, 25, 23

Sutton, E. C., Peng, R., Danchi, W. C., et al. 1995, ApJS, 97, 455

Tielens, A. G. G. M. 1983, A\&A, 119, 177

Turner, B. E. 1990, ApJ, 362, L29

Turner, B. E. 2001, ApJS, 136, 579

van der Tak, F. F. S., van Dishoeck, E. F., \& Caselli, P. 2000a, A\&A, 361,327

van der Tak, F. F. S., van Dishoeck, E. F., Evans, N. J., Bakker, E. J., \& Blake, G. A. 1999, ApJ, 522, 991

van der Tak, F. F. S., van Dishoeck, E. F., Evans, N. J., \& Blake, G. A. 2000b, ApJ, 537, 283

van Dishoeck, E. F., \& Blake, G. A. 1998, ARA\&A, 36, 317

van Dishoeck, E. F., Blake, G. A., Jansen, D. J., \& Groesbeck, T. D. 1995, ApJ, 447, 760

van Dishoeck, E. F., Jansen, D. J., \& Phillips, T. G. 1993, A\&A, 279, 541

van Zadelhoff, G.-J., Dullemond, C. P., Yates, J. A., et al. 2002, A\&A, submitted

Viti, S., Caselli, P., Hartquist, T. W., \& Williams, D. A. 2001, A\&A, 370, 1017

Walker, C. K., Adams, F. C., \& Lada, C. J. 1990, ApJ, 349, 515

Walker, C. K., Lada, C. J., Young, E. T., Maloney, P. R., \& Wilking, B. A. 1986, ApJ, 309, L47

Walker, C. K., Lada, C. J., Young, E. T., \& Margulis, M. 1988, ApJ, 332, 335

Walmsley, C. M. 1992, in Chemistry and Spectroscopy of Interstellar Molecules, ed. D. K. Bohme, et al. (Univ. of Tokyo), 267

Walmsley, C. M., \& Schilke, P. 1993, in Dust and Chemistry, ed. T. J. Millar, \& D. A. Williams (Kluwer, Dordrecht), 37

Whittet, D. C. B. 1974, MNRAS, 168, 371

Wilson, T. L., \& Rood, R. 1994, ARA\&A, 32, 191

Wootten, A. 1989, ApJ, 337, 858

Wootten, A., \& Loren, R. B. 1987, ApJ, 317, 220

Zhou, S. 1995, ApJ, 442, 685 